\title{
Dual targeting of the DNA damage response pathway and BCL- 2 in diffuse large B-cell lymphoma
}

\author{
Alessandra Rossi ${ }^{1}$, Stefania Orecchioni ${ }^{2}$, Paolo Falvo ${ }^{2}$, Valentina Tabanelli ${ }^{3}$, Elena Baiardi $\mathbb{D}^{1}$, Claudio Agostinelli ${ }^{4,5}$, Federica Melle ${ }^{3}$, \\ Giovanna Motta ${ }^{3}$, Angelica Calleri ${ }^{3}$, Stefano Fiori ${ }^{3}$, Chiara Corsini ${ }^{2}$, Beatrice Casadei ${ }^{6}$, Saveria Mazzara ${ }^{3}$, Umberto Vitolo ID $^{7}$, \\ Francesco Bertolini ${ }^{2}$, Pier Luigi Zinzani (iD ${ }^{6}$, Myriam Alcalay ${ }^{8,9}$, Pier Giuseppe Pelicci ${ }^{8,9}$, Stefano Pileri ${ }^{3}$, Corrado Tarella (iD ${ }^{1,10^{凶}}$ and \\ Enrico Derenzini (iD ${ }^{1,10 凶}$
}

(c) The Author(s) 2021

Standard chemotherapies for diffuse large B-cell lymphoma (DLBCL), based on the induction of exogenous DNA damage and oxidative stress, are often less effective in the presence of increased MYC and BCL-2 levels, especially in the case of double hit (DH) lymphomas harboring rearrangements of the $M Y C$ and $B C L-2$ oncogenes, which enrich for a patient's population characterized by refractoriness to anthracycline-based chemotherapy. Here we hypothesized that adaptive mechanisms to MYC-induced replicative and oxidative stress, consisting in DNA damage response (DDR) activation and BCL-2 overexpression, could represent the biologic basis of the poor prognosis and chemoresistance observed in $M Y C / B C L$-2-positive lymphoma. We first integrated targeted gene expression profiling (T-GEP), fluorescence in situ hybridization (FISH) analysis, and characterization of replicative and oxidative stress biomarkers in two independent DLBCL cohorts. The presence of oxidative DNA damage biomarkers identified a poor prognosis double expresser (DE)-DLBCL subset, characterized by relatively higher $B C L-2$ gene expression levels and enrichment for DH lymphomas. Based on these findings, we tested therapeutic strategies based on combined DDR and BCL-2 inhibition, confirming efficacy and synergistic interactions in in vitro and in vivo DH-DLBCL models. These data provide the rationale for precision-therapy strategies based on combined DDR and BCL-2 inhibition in DH or DE-DLBCL.

Leukemia (2022) 36:197-209; https://doi.org/10.1038/s41375-021-01347-6

\section{INTRODUCTION}

Diffuse Large B-cell lymphoma (DLBCL) is the most common nonHodgkin lymphoma (NHL) subtype, and yet $40 \%$ of patients are resistant to current therapies [1-3], which are still based on the induction of exogenous DNA damage with anthracycline-based chemotherapy regimens representing the standard of care [1-3]. The cell of origin (COO) determined by gene expression profiling (GEP) is a well-established prognostic predictor in DLBCL [4-7]. In general, DLBCLs with a GEP signature related to activated B-cell cells ( $A B C$ subgroup) have a worse outcome compared to their germinal center B-cell (GCB) counterparts [4-7], and display higher expression levels of MYC and BCL2 [8] and oncogenic addiction to nuclear factor kappa-B (NF-kB) signaling [9]. However, despite its prognostic relevance, COO-based precision therapy has not yet translated into meaningful clinical benefits [10-12]. Besides the
$\mathrm{COO}$, overexpression or genomic rearrangements of the $M Y C$ and $B C L-2$ oncogenes are powerful negative prognostic factors in DLBCL $[13,14]$. Due to the fact that concurrent MYC and BCL-2 rearrangements enrich for a patient's population characterized by refractoriness to standard anthracycline-based chemotherapy, these lymphomas are now classified as a separate disease entity (HG-BCL w/DH) [15] and currently treated with more intensive chemotherapy regimens, representing a major unmet need in lymphoma therapy $[16,17]$. On the other hand, the observation that a fraction of HG-BCL w/DH can be cured with standard therapies underlines the concept that the mechanisms underlying chemoresistance in $\mathrm{MYC} / \mathrm{BCL}-2$ positive $\mathrm{DLBCL}$ are still poorly defined. Recent evidence suggests that MYC-positive tumors are characterized by replicative and oxidative stress leading to inherent DNA damage and genomic instability [18-20]. Constitu-

\footnotetext{
'Onco-Hematology Division, IEO European Institute of Oncology IRCCS, Milan, Italy. ${ }^{2}$ Laboratory of Hematology-Oncology, IEO European Institute of Oncology IRCCS, Milan, Italy. ${ }^{3}$ Division of diagnostic Haematopathology, IEO European Institute of Oncology IRCCS, Milan, Italy. ${ }^{4}$ Hematopathology Unit, Department of Experimental, Diagnostic, and Specialty Medicine (DIMES), Bologna University School of Medicine, Bologna, Italy. ${ }^{5}$ Haematopathology Unit, IRCCS Azienda Ospedaliero-Universitaria di Bologna, Bologna, Italy. ${ }^{6}$ IRCCS Azienda Ospedaliero-Universitaria di Bologna, Institute of Hematology and Medical Oncology "L. e A. Seragnoli", Department of Experimental, Diagnostic, and Specialty Medicine (DIMES), University of Bologna, Bologna, Italy. ${ }^{7}$ Multidisciplinary Oncology Outpatient Clinic, Candiolo Cancer Institute, FPO-IRCCS, Candiolo, Italy. ${ }^{8}$ Department of Experimental Oncology, IEO European Institute of Oncology IRCCS, Milan, Italy. ${ }^{9}$ Department of Oncology and Hemato-Oncology, University of Milan, Milan, Italy. ${ }^{10}$ Department of Health Sciences, University of Milan, Milan, Italy. ${ }^{凶}$ email: corrado.tarella@unimi.it; enrico.derenzini@ieo.it
}

Received: 1 November 2020 Revised: 28 June 2021 Accepted: 8 July 2021

Published online: 24 July 2021 
Table 1. Patients characteristics.

\begin{tabular}{|c|c|c|c|c|}
\hline Factor & $\begin{array}{l}\text { DLCL04 } \\
(N=69)\end{array}$ & $\begin{array}{l}\text { Real life } \\
(n=66)\end{array}$ & $p$ value & $\begin{array}{l}\text { Combined } \\
(n=135)\end{array}$ \\
\hline \multicolumn{5}{|l|}{$\mathrm{COO}$} \\
\hline$A B C$ & 21 & 12 & 0.1 & 33 \\
\hline GCB/UNCL & 48 & 54 & & 102 \\
\hline Age & $51(18-63)$ & $\begin{array}{l}64 \\
(17-85)\end{array}$ & $<0.01$ & $57(17-85)$ \\
\hline \multicolumn{5}{|l|}{ IPI } \\
\hline $0-1$ & - & 17 & 0.2 & 17 \\
\hline $2-3$ & 57 & 45 & & 102 \\
\hline $4-5$ & 12 & 4 & & 16 \\
\hline \multicolumn{5}{|l|}{ Treatment } \\
\hline R-CHOP-like & 39 & 66 & - & 105 \\
\hline $\begin{array}{l}\text { R-CHOP- } \\
\text { like }+ \text { ASCT }\end{array}$ & 30 & - & & 30 \\
\hline \multicolumn{5}{|c|}{ MYC/BCL-2 status } \\
\hline \multicolumn{5}{|l|}{ Nano } \\
\hline DE & 18 & 17 & 0.9 & 35 \\
\hline Non-DE & 51 & 49 & & 100 \\
\hline $\mathrm{DH}$ & 4 & 3 & 0.9 & 7 \\
\hline \multicolumn{5}{|l|}{$\gamma \mathrm{H} 2 \mathrm{AX} / 8-\mathrm{OHDG}$} \\
\hline $\mathrm{DE}$ & 38 & 31 & 0.39 & 69 \\
\hline NON DE & 31 & 35 & & 66 \\
\hline
\end{tabular}

COO Cell of origin, IPI international prognostic index, DH double hit, ASCT autologous stem cell transplant, nano NanoString, $D E$ double expresser, $A B C$ activated B-cell, GCB/UNCL germinal center B-cell/unclassified. tive activation of the DNA damage response (DDR) pathway is one of the main mechanisms by which cancer cells cope with replicative stress, avoiding intolerable levels of endogenous DNA damage [21-23]. On the other hand, it is well known that BCL-2 overexpression synergizes with MYC in driving B-cell lymphomagenesis, by counteracting MYC-related proapoptotic effects and oxidative stress [24-28]. Of note, constitutive DDR activation correlates with MYC levels predicting poor prognosis [29], and therapeutic approaches targeting DDR through inhibition of the Ataxia teleangiectasia and Rad3 related (ATR)-checkpoint kinase 1/ $2($ CHK1/2) axis showed efficacy in preclinical models of MYCpositive DLBCL, including those with TP53 mutations/deletions and CDKN2A loss which are mechanistically linked to anthracycline resistance [30-33].

Since chemotherapy exerts its cytotoxic effects through exogenous DNA damage and induction of reactive oxygen species (ROS), adaptive mechanisms to replicative and oxidative stress (consisting in DDR activation and upregulation of antioxidant capacity), could represent the biologic basis of the poor prognosis and chemoresistance observed in $\mathrm{MYC} / \mathrm{BCL}$-2-double expresser DLBCL and HG-BCL w/DH.

In an effort to design specific therapies for MYC/BCL-2 positive DLBCL, we first integrated targeted-GEP (T-GEP), fluorescence in situ hybridization (FISH) analysis, and functional characterization of replicative and oxidative stress biomarkers in two independent DLBCL cohorts. Since the presence of oxidative DNA damage biomarkers identified a poor prognosis DE-DLBCL subset, which was characterized by relatively higher $B C L-2$ gene expression levels and enrichment in HG-BCL $w / D H$, we then tested therapeutic strategies based on combined DDR and BCL-2 inhibition, confirming efficacy and synergistic interactions in in vitro and in vivo HG-BCL w/DH models. These data provide the rationale for novel precision therapy strategies based on combined DDR and BCL-2 inhibition in double-hit DLBCL.

\section{DLCL04 Study}

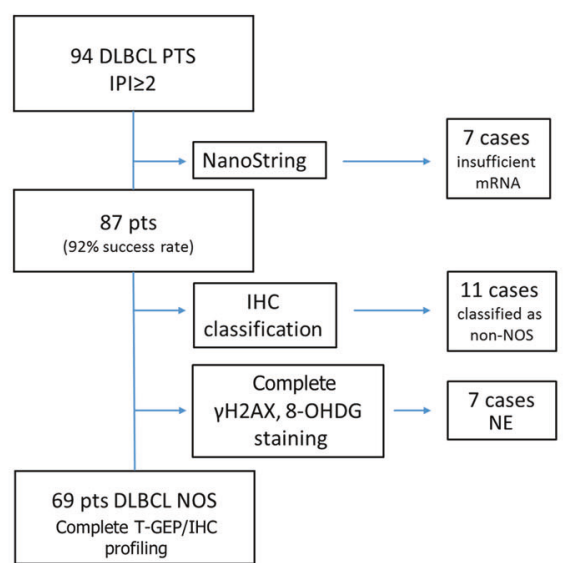

\section{Real Life Cohort}

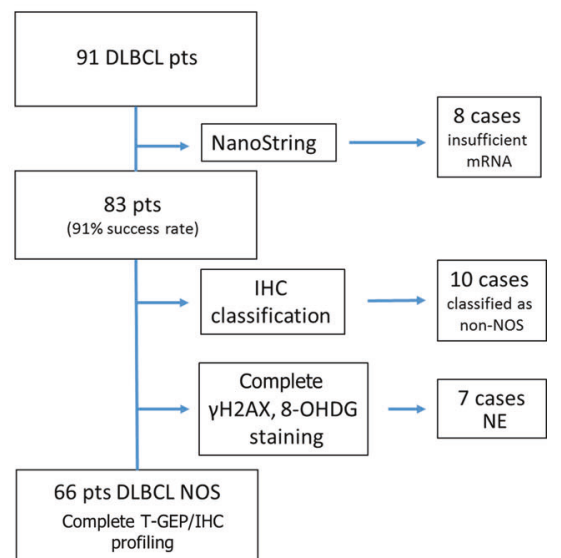

Fig. 1 Study cohorts. A On the left, the discovery cohort is represented. 94 stage III-IV DLBCL patients enrolled in the DLCL04 trial with available FFPE tissue were initially considered in this analysis. T-GEP success rate was $92.6 \%(n=87)$, with seven cases not yielding enough high-quality mRNA to undergo successful GEP assessment. Only cases of non-otherwise specified (NOS) histology (including those originally diagnosed as DLBCL-NOS and nowadays included in the HG-BCL provisional category) were considered; therefore 11 cases classified in different DLBCL categories were excluded. In seven cases that were not evaluable (NE), we could not retrieve enough tissue for additional immunohistochemistry studies and these cases were excluded. Sixty-nine NOS-DLBCL FFPE patient samples from the DLC04 trial were finally included in this study. B On the right a "real-life" validation cohort including 66 consecutive DLBCL NOS cases with available FFPE tissue for TGEP and IHC, treated with R-CHOP/CHOP-like regimens. Success rates of T-GEP, and cases excluded for histologic classification or IHC tissue availability issues are detailed in the figure. 
A
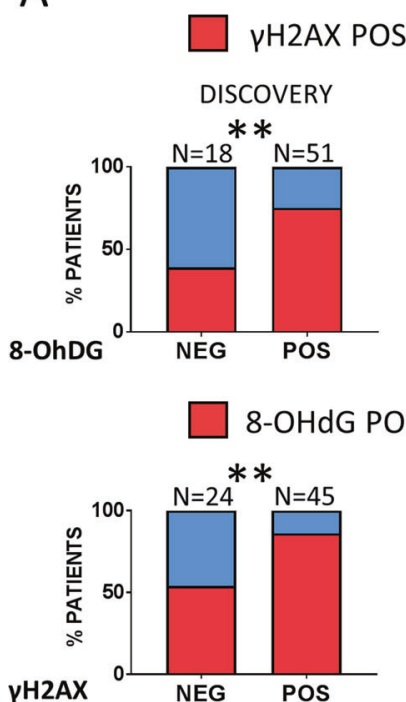

B

$\mathrm{H} 2 \mathrm{AX}$ NEG

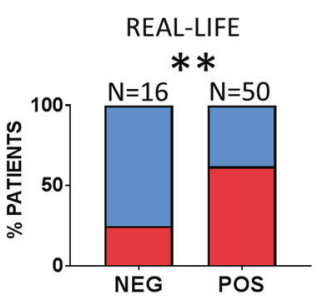

8-OHdG NEG

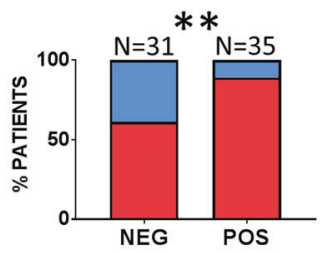

8-OHdG

YH2AX

S139
C

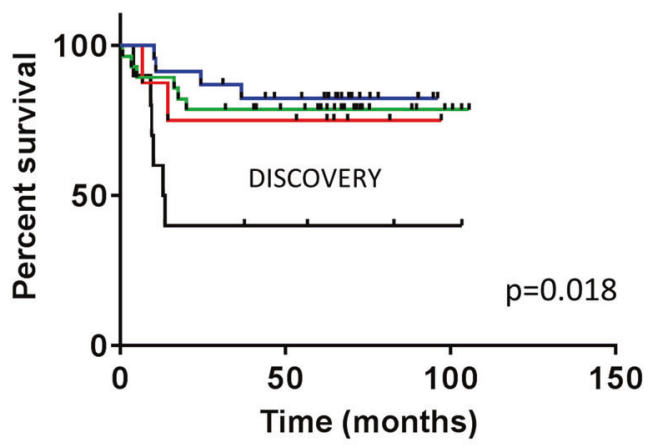

E

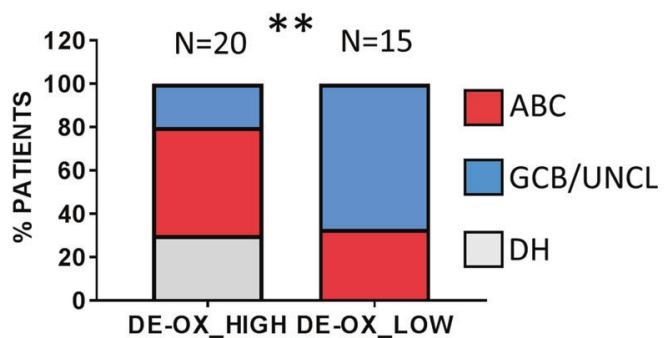

F

D
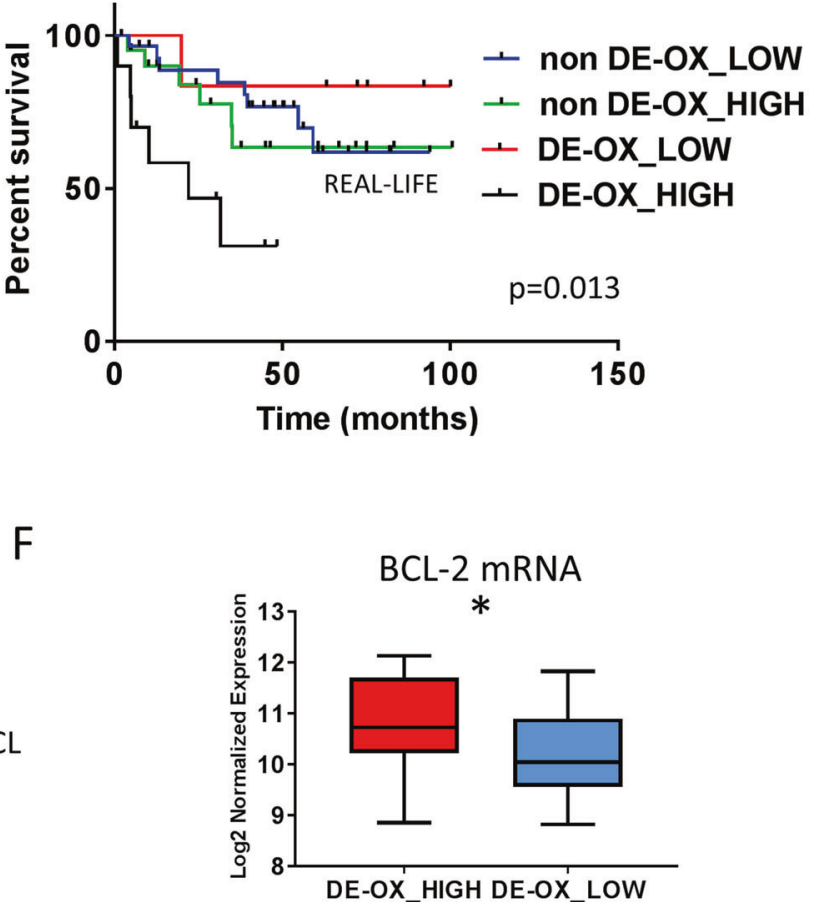

Fig. 2 Oxidative and replicative stress biomarkers in poor prognosis DLBCL subsets. A In the upper panel, bar graph indicating the proportion of 8-OhDG positive and negative cases according to the expression levels of $\gamma \mathrm{H} 2 \mathrm{AX}$ in the discovery (left) and real-life cohort (right). In the lower panel, the proportion of $\gamma \mathrm{H} 2 \mathrm{AX}$ positive and negative cases according to the expression levels of 8-OhDG in the discovery (left) and real-life cohort (right). $P$ value was calculated with the chi-square test. A $p$ value $<0.05$ was considered as statistically significant: ${ }^{*}$ $<0.05, * *<0.01$. POS (positive); NEG (negative). See also Figure S1. B Representative examples of 8-OHdG and $\gamma \mathrm{H} 2 \mathrm{AX}$ positive (right panel) and negative (left panel) DLBCLs. Original magnification, 600x. C OS of the discovery cohort (DLCL04; $n=69$ ) according to the MYC/BCL-2 status as determined by T-GEP and to $\gamma \mathrm{H} 2 \mathrm{AX} / 8-\mathrm{OhDG}$ levels as assessed by IHC. DLBCL showing increased levels of $M Y C$ and $B C L-2 \mathrm{mRNA}$ by T-GEP (MYC/BCL-2 DE) and concurrent overexpression of $\gamma \mathrm{H} 2 \mathrm{AX}$ and 8-OhDG (DE-OX_high subgroup) are characterized by a worse outcome compared to all other subgroups. $P$ values were calculated with the log-rank test. See also Figures S2 and S3. D OS of the real-life validation cohort $(n=66)$ according to the MYC/BCL-2 status as determined by T-GEP and to $\gamma \mathrm{H} 2 \mathrm{AX} / 8-\mathrm{OhDG}$ levels as assessed by IHC. DLBCL showing increased levels of MYC and BCL-2 mRNA by T-GEP (MYC/BCL-2 DE) and concurrent overexpression of $\gamma \mathrm{H} 2 \mathrm{AX}$ and 8-OhDG (DE-OX_high subgroup) are characterized by a worse outcome compared to all other subgroups. $P$ values were calculated with the log rank test. See also Figure S3. E Bar graphs representing the proportions of different DLBCL subsets in the DE-OX_high and DE-OX_low subgroups (analysis of the whole cohort: discovery + validation), showing relative increase in ABC cases and significant enrichment HG-BCL w/DH in the DE-OX_high subgroup. See also Table 2 for detailed statistics. See also Figure S3. F Box plot graph showing BCL-2 mRNA levels as assessed by T-GEP in the DE-OX_high vs DE-OX_low subgroup. Differences between groups were calculated with the Student $T$ test. ${ }^{*} p<0.05,{ }^{* *} p<0.01$. 
Table 2. Characteristics of the DE-OX_low and DE-OX-high subgroups (analysis of the whole cohort: discovery + validation).

\begin{tabular}{|llll|}
\hline & $\begin{array}{l}\text { DE-OX_low } \\
(\boldsymbol{n}=\mathbf{1 5})\end{array}$ & $\begin{array}{l}\text { DE-OX_high } \\
(\boldsymbol{n}=\mathbf{2 0})\end{array}$ & $\boldsymbol{p}$ value \\
\hline Treatment & & & \\
\hline R-CHOP_like & 10 & 13 & 0.99 \\
\hline R-CHOP_like + ASCT & 5 & 7 & \\
\hline COO & & & 0.99 \\
\hline GCB & 5 & 6 & 0.45 \\
\hline UNCL & 5 & 4 & 0.49 \\
\hline ABC & 5 & 10 & 0.027 \\
\hline DH & & 6 & 0.013 \\
\hline DH + ABC & 0 & 16 & \\
\hline
\end{tabular}

$C O O$ cell of origin, $D H$ double hit, $A S C T$ autologous stem cell transplant, $D E$ double expresser, $A B C$ activated B-cell, GCB/UNCL germinal center B-cell/ unclassified.

$P$ value was calculated with the Fisher's exact test.

${ }^{a}$ The remaining $1 \mathrm{DH}$ case clustered in the non-DE subset. All DH cases were of GCB origin.

\section{METHODS \\ Patients}

In the present study, we analyzed two independent patients cohorts: 69 patients from the DLCL04 study [34], a prospective randomized phase 3 clinical trial investigating the role of first-line autologous stem cell transplant (ASCT) consolidation after chemoimmunotherapy in $\mathrm{CD} 20+$ $\mathrm{DLBCL}$, and 66 patients from a real-life cohort treated with R-CHOP/CHOPlike regimens at S. Orsola-Malpighi Hospital, Bologna (Italy), from 2007 to 2012. Patients characteristics are summarized in Table 1.

The study flowchart is depicted in Fig. 1 and additional details are provided in supplement.

This study was approved by the Institutional Review Boards of the participating centers, in accordance with the Declaration of Helsinki.

\section{Targeted gene expression profiling (T-GEP) panel}

Gene expression was measured on the NanoString nCounter Analysis System (NanoString Technologies, Seattle, WA, USA). The original T-GEP panel contains 22 genes: 15 genes used to assign COO subtype [6], 5 housekeeping genes (UBXN4, ISY1, R3HDM1, WDR55, TRIM56); and the additional genes of interest: $M Y C$ and $B C L-2$. The complete list of genes, target sequences, and detailed methods are available in supplement.

\section{Immunohistochemistry and fluorescence in situ hybridization (FISH)}

Immunohistochemistry $(\mathrm{IHC})$ was centralized in Milan for the DLCL04 trial and in Bologna for the real-life control group. Primary antibody source and dilutions are shown in Table S1 and a detailed description of IHC and FISH methods is provided in supplement.

The cut-off values of $50 \%$ and $40 \%$ positive neoplastic cells were applied for BCL2 and MYC respectively [14], and 50\% for $\mathrm{\gamma H} 2 \mathrm{AX}$ and $8-\mathrm{OHdG}$.

\section{Reagents, in vitro assays, and cell lines}

Prexasertib was provided by the Eli Lilly company for in vitro studies, and was purchased from Selleckchem (Houston, Tx) for in vivo studies. AZD7762, MK-8776, Venetoclax and the MCL-1 inhibitor S63845 were purchased from Selleckchem (Houston, TX).

Detailed information on cell lines, 8-OHdG ELISA assay, Caspase 3/7 assay, cell cycle analyses, qPCR assays, proliferation assays (Cell Titer Glo, Promega), and western blot antibodies are provided in supplement. The Tet-OFF MYC P-4936 cell line $[35,36]$, which carries a conditional, tetracycline-regulated MYC promoter, was provided by Dr. A. Younes lab (Memorial Sloan Kettering Cancer Center, New York, NY). Inducible overexpression of $B C L 2$ in the SUDHL5 (BCL2 negative) cell line was performed by using the Cellecta InDOXible Tet-Activated CDNA Lentiviral Expression System (custom Cellecta). Detailed methods for in vitro studies are provided in supplement.

\section{High-throughput screening experiments}

High-throughput drug screening experiments were performed as previously described [37]. Plates were imaged after incubation with Alamar Blue on the LEADseeker ${ }^{\mathrm{TM}}$ Multimodality Imaging System (GE Healthcare, Piscataway, NJ). In order to evaluate synergy, we compared the observed activity to the expected activity of the combination at that dose level under Bliss independence model [38, 39]. HTS experiments and analyses were performed at the Memorial Sloan Kettering Cancer Center, New York. Detailed information is available in supplement.

\section{In vivo studies}

Experiments involving animals were approved by the Italian Ministry of Health and have been performed in accordance with the applicable Italian laws (D.L.vo 26/14 and following amendments), the Institutional Animal Care and Use Committee, the institutional guidelines of the European Institute of Oncology and the ARRIVE guidelines [40].

The luciferase-expressing patient-derived xenograft (PDX) line DFBL69487-V3-mCLP [41] was obtained from the Public Repository of Xenografts (www.proxe.org). Detailed information is available in supplement.

\section{Statistical analysis}

Survival data and correlations were analyzed retrospectively. For survival analysis, we used the Kaplan-Meier method [42] to estimate overall survival (OS). The two-tailed Student $t$ test and Wilcoxon Rank test were used to estimate statistical significance. Correlations and differences in patients' characteristics were analyzed with the chi-square and Fisher's exact test. The PRISM software was used for the statistical analyses (v7). Significance was set at $P<0.05$. Combination index analysis was performed using the Chou-Talalay method [43].

T-GEP statistical analyses were calculated with the R software (v3.5.0) [44]. A detailed description is provided in supplement.

\section{RESULTS}

Replicative and oxidative stress biomarkers identify poor prognosis subsets of double expresser DLBCL

In order to investigate the relationship between COO classification, MYC/BCL-2 status, and replicative/oxidative stress biomarkers, we first profiled two independent case series of chemoimmunotherapy-treated DLBCL with T-GEP, FISH, and immunohistochemistry: a discovery cohort from the DLC04 study [34], and a validation cohort of patients treated in real-life clinical practice. Patients characteristics are shown in Table 1 and were similar in the two cohorts (except for median age, significantly higher in the real-life cohort), with no significant differences in the overall outcome (Figure S1). For T-GEP studies, we used a digital multiplex gene expression profiling platform (NanoString Technology) with a panel containing 22 genes (15 genes for COO subtyping according to LST algorithm [6] plus MYC and $B C L 2$ and 5 housekeeping genes). T-GEP, FISH and immunohistochemistry (IHC) profiling for C-MYC, BCL-2, the phosphorylated form of $\mathrm{H} 2 \mathrm{AX}$ at $\mathrm{S} 139(\mathrm{\gamma H} 2 \mathrm{AX}$, a biomarker of DNA damage and DDR activation) $[45,46]$ and 8-hydroxy-2'-deoxyguanosine (8OHdG, an oxidative DNA damage marker) [47] were available in 69 patients from the DLC04 study [34], and 66 patients treated in the real-life cohort (Fig. 1 A, B).

The two cohorts displayed similar patterns of $\mathrm{\gamma H} 2 \mathrm{AX}$ and 8OHdG expression (cut-off value $50 \%$ of positive cells, with $55 \%$ and $47 \%$ of cases showing dual nuclear positivity for $\mathrm{\gamma H} 2 \mathrm{AX}$ and 8OHdG in the DLCL04 and real-life cohort respectively). The expression levels of $\mathrm{\gamma H} 2 \mathrm{AX}$ and $8-\mathrm{OHdG}$ were significantly correlated, with the $8-\mathrm{OHdG}$ positive subgroup showing a significantly higher fraction of $\mathrm{\gamma H} 2 \mathrm{AX}$ positive cases, as compared to the $8-\mathrm{OHdG}$ negative subset. In line with this observation, the proportion of 8-OhDG-positive samples was significantly increased in the $\mathrm{yH} 2 \mathrm{AX}$-positive subset in both cohorts (Fig. $2 \mathrm{~A}, \mathrm{~B}$ ).

In order to define the prognostic implications of $\mathrm{\gamma H} 2 \mathrm{AX}$ and 8OHdG expression with respect to the $\mathrm{COO}$ and MYC/BCL-2 status, 
A

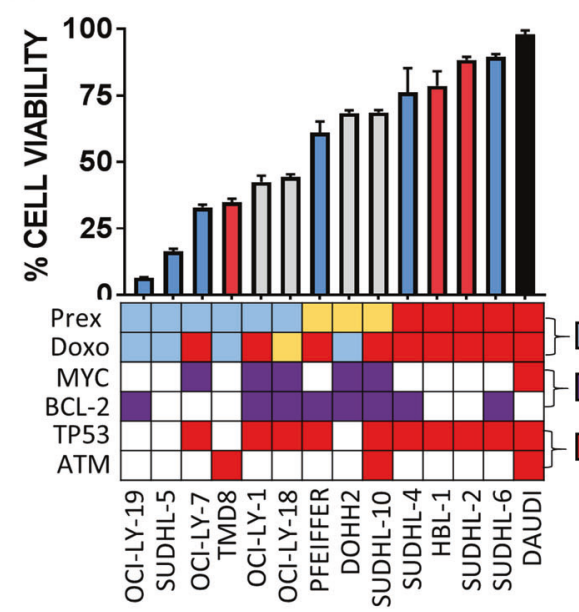

C

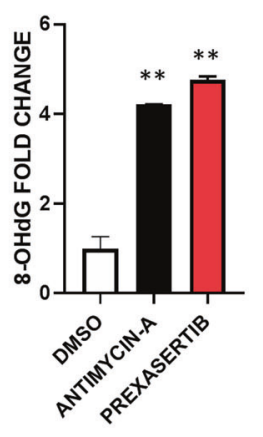

$E$

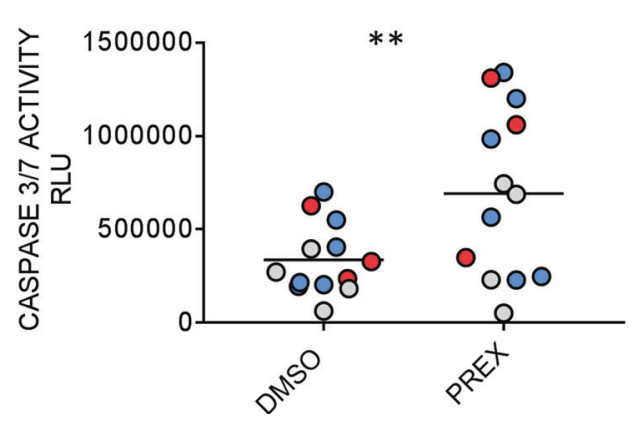

we analyzed the impact of these variables on OS rates in the two cohorts.

Although there was a significant correlation between MYC and $B C L-2$ mRNA (assessed with T-GEP) and protein levels (assessed by $\mathrm{IHC}$ ) (Figure S2A), MYC/BCL-2 status evaluated by T-GEP outperformed IHC for prognostic stratification in both cohorts (Table S2 and Figure S2B) (patients were classified as high and low MYC or BCL-2 expressers based on the median normalized $M Y C$ and $B C L-2$ mRNA levels in the respective cohorts).

In both patients cohorts, dual nuclear positivity for $\mathrm{YH} 2 \mathrm{AX}$ and 8-OHdG identified a MYC/BCL-2 mRNA Double Expresser (DE)-
B

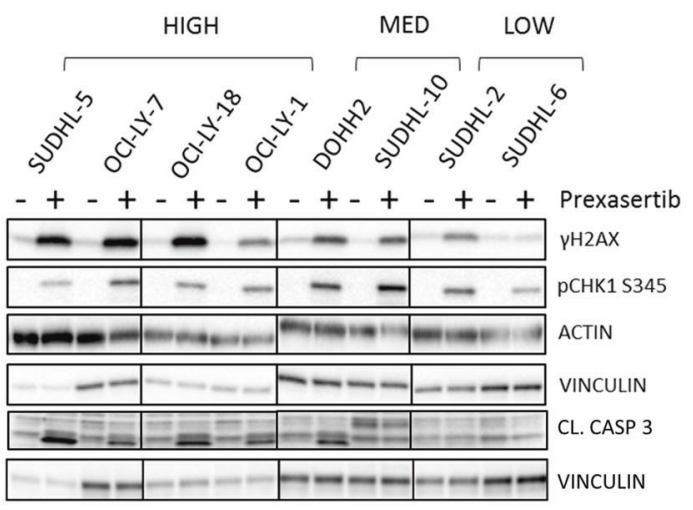

D

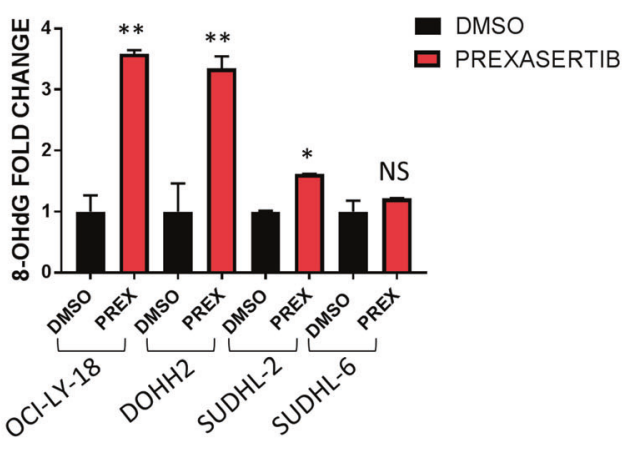

$\mathrm{F}$

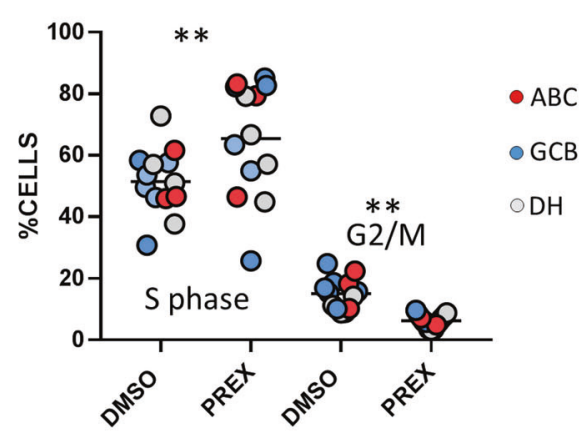

DLBCL subset characterized by dismal outcome (hereafter defined as DE-OX_high) (Fig. 2 C, D). Interestingly MYC/BCL-2 mRNA DE cases with low oxidative DNA damage (DE-OX_low) had a very favorable outcome, with OS rates comparable to non-DE cases (Fig. 2 C, D). Similar trends were observed in both cohorts (Fig. 2 C, D) and a cumulative analysis ( $n=135$ patients) confirmed a significantly worse OS rate for the DE-OX_high subgroup, compared to all other subgroups (Figure S3A).

To further define the biologic characteristics of the DE-OX_high subgroup we investigated COO subtyping and the presence of $M Y C$ and $B C L-2$ rearrangements (HG-BCL w/DH) across different 
Fig. 3 DDR inhibition with Prexasertib determines oxidative DNA damage accumulation and apoptosis in DLBCL cell lines. A Bar graph showing percentages of cell viability measured with the Cell Titer Glo assay (Promega) in 13 DLBCL cell lines and $1 \mathrm{BL}$ cell line (Daudi) treated with Prexasertib $125 \mathrm{nM}$ for $24 \mathrm{~h}$. Below the graph, a heat map showing: 1- information on Prexasertib (Prex) and Doxorubicin (Doxo) sensitivity defined as high $>50 \%$ inhibition; med $25-50 \%$ inhibition; low $<25 \%$ inhibition. 2- annotation regarding MYC, BCL-2, TP53, ATM genomic alteration status in all cell lines (rearr.: rearrangement; wt: wild type; mut/del: mutation/deletion). Detailed reference of genomic data annotation is available in supplement. Error bars represent the standard error of the mean (SEM) of triplicate experiments. See also Figure S4. B Representative western blot assays depicting the effects of $125 \mathrm{nM}$ Prexasertib on expression levels of $\gamma \mathrm{H} 2 \mathrm{AX}, \mathrm{pCHK} 1 \mathrm{~S} 345$, Caspase 3 cleavage after $6 \mathrm{~h}$ of incubation in DLBCL cell lines characterized by high, intermediate (med), and low sensitivity to Prexasertib. C Bar graph showing 8-OhDG levels in DNA extracted from DOHH2 cells treated with DMSO or Prexasertib ( $250 \mathrm{nM})$ and Antimycin A (100 nM) for $6 \mathrm{~h}$. Error bars represent standard error of the mean (SEM) of triplicate experiments. Differences between groups were calculated with the Student $T$ test. ${ }^{*} p<0.05,{ }^{* *} p<0.01$. D Bar graph showing 8-OhDG levels in DNA extracted from OCI-Ly-18, DOHH2, SUDHL-2, and SUDHL-6 cells treated with DMSO or Prexasertib (125 nM) (PREX) for $6 \mathrm{~h}$. Error bars represent standard error of the mean (SEM) of triplicate experiments. Differences between groups were calculated with the Student $T$ test. ${ }^{*} p<0.05,{ }^{* *} p<0.01$. See also Figure S4. E Scatter plot representing the effects of 125 $\mathrm{nM}$ Prexasertib (PREX) on apoptosis in DLBCL cell lines. Cells were incubated with Prexasertib for $24 \mathrm{~h}$ and caspase $3 / 7$ activation assessed with the Caspase Glo 3/7 assay system (Promega ${ }^{T M}$ ). Each point represents the mean of three independent experiments. $P$ value was calculated with the paired Student's $T$ test. See also Figure S4 and S5. RLU (relative light units). F Scatter plot representing the effects of $125 \mathrm{nM}$ Prexasertib (PREX) on cell cycle in DLBCL cell lines, showing significant accumulation of DLBCL cells in the $S$ phase and reduction of the G2/M phase. Cells were incubated with Prexasertib for $24 \mathrm{~h}$ and changes in cell cycle fractions were assessed by flow cytometry with propidium iodide staining. Each point represents the mean of three independent experiments. $P$ value was calculated with the paired Student's $T$ test. See also Figure S5.

patients' subsets (DE-OX_high vs DE-OX_low). Interestingly we found that the DE-OX_high subgroups were enriched in HG-BCLs w/DH (Fig. 2E), showed a relative increase in $A B C$ cases, and were characterized by relatively higher $B C L-2$ mRNA levels compared to the DE-OX_low subsets (Fig. 2F). Notably, all but one HG-BCL w/ $\mathrm{DH}$ clustered in the DE-OX_high subgroups (Table 2). The outcome of DE-OX_HIGH subset was dismal irrespective of the $\mathrm{DH}$ status, with similarly poor OS rates observed in $\mathrm{DH}$ and non$\mathrm{DH}$ cases (Figure S3B). These data indicate that the expression of replicative and oxidative stress biomarkers such as $\mathrm{yH} 2 \mathrm{AX}$ and 8OHdG could define a subset of $M Y C / B C L-2$ DE DLBCL with specific molecular features and characterized by a worse outcome.

\section{Functional characterization of replicative and oxidative stress biomarkers following DDR inhibition in DLBCL}

To further investigate these findings we treated a panel of DLBCL cell lines with the CHK1/2 inhibitor Prexasertib [48], and assessed the effects of treatment on $\mathrm{\gamma H} 2 \mathrm{AX}$ and $8-\mathrm{OHdG}$ levels. Prexasertib showed antiproliferative activity at sub-micromolar concentrations across multiple cell lines, irrespective of the cell doubling time, COO, MYC/BCL-2 rearrangements, TP53, and (Ataxia Telangiectasia Mutated) ATM status (Fig. 3A and Figure S4A,B). As opposite, the in vitro efficacy of the DNA-damaging agent Doxorubicin was closely related to the TP53 wild-type status (Figure S4C). As previously reported $[48,49]$, treatment with Prexasertib resulted in reduced clearance of DNA damage foci and consequent upstream DDR activation as demonstrated by increased $\mathrm{yH} 2 \mathrm{AX}$ and $\mathrm{p}-\mathrm{CHK} 1$ S345 levels respectively (Fig. 3B). While in some cell lines Prexasertib and Doxorubicin had similar effects on cell viability and DNA damage accumulation, DDR inhibition by Prexasertib significantly increased $\mathrm{YH} 2 \mathrm{AX}$ levels in cell lines where Doxorubicin failed to determine significant DNA damage (Figure S4D). Furthermore, Prexasertib-induced DDR inhibition resulted in increased levels of DNA oxidation as assessed with a 8-OHdG ELISA assay (Fig. 3C,D). The extent of $8-\mathrm{OHdG}$ induction was similar to that observed after treatment with known oxidative stress-inducing agents (Fig. 3C), such as Antimycin A [50], and was more prominent in Prexasertib-sensitive cell lines (Fig. 3D), showing a dose-dependent pattern (Figure S4E). Prexasertib treatment induced significant apoptosis in DLBCL cell lines irrespective of TP53 status and was associated with significant accumulation of cells in $S$ phase of the cell cycle, in line with previous observations [51] (Fig. 3E, F and Figures S4F and S5A-F). These data, together with our observations on $\mathrm{yH} 2 \mathrm{AX}$ and $8-\mathrm{OHdG}$ expression patterns in DLBCL tissues, suggest that oxidative stress could be a major source of inherent DNA damage contributing to constitutive DDR activation in DLBCL, and indicate that DDR inhibition induces oxidative DNA damage accumulation, cell cycle arrest and apoptosis in DLBCL cell lines.

\section{BCL-2 inhibition enhances the activity of DDR inhibitors in in vitro DLBCL models}

Since constitutive expression of replicative and oxidative stress biomarkers defined a subset of DE-DLBCL characterized by worse outcome and increased $B C L-2$ gene expression levels, we next investigated the therapeutic implications of these findings in in vitro DLBCL models, by combining different DDR inhibitors with the selective $B C L-2$ inhibitor Venetoclax. In a preliminary experiment, using a combinatorial high-throughput drug screening (HTS) approach we first combined two DDR inhibitors (the CHK1/ CHK2 inhibitor AZD-7762 and the selective CHK1 inhibitor MK8776 ) with the BCL-2 inhibitor Venetoclax in $10 \mathrm{DLBCL}$ cell lines. As shown in Fig. $4 \mathrm{~A}, \mathrm{BCL}-2$ inhibition significantly enhanced the antiproliferative activity of $\mathrm{CHK}$ inhibitors in $\mathrm{BCL}-2$ positive cell lines ( $A B C$-derived or GCB-derived harboring $B C L-2$ rearrangements, Figure S6 A-D). In general, better trends were observed with CHK1/2 inhibition (AZD-7762) than with selective CHK1 inhibition (MK-8776) (Fig. 4A). BCL-2 protein expression data and HTS results of individual cell lines are shown in supplement (Figure S6 A-E). Individual matrices of 1 representative GCB cell line with $B C L-2$ translocation (SUDHL-4) and $1 A B C$-derived cell line $(\mathrm{HBL}-1)$ treated with AZD7762 are represented in Fig. 4B (complete data are shown in HTS data supplement). Since we described a significant enrichment of HG-BCL $w / D H$ cases in the DE-Ox high subset, we then extended our cell line panel including different DH DLBCL models, and validated HTS results by cell-titerglo assay using the CHK1/2 inhibitor Prexasertib. As observed with AZD-7762, BCL-2 inhibition significantly enhanced the efficacy of Prexasertib in multiple DLBCL cell lines (Fig. 4C,D and Figure S7). In line with our HTS data, the most favorable interactions were observed in $B C L-2$ positive $D L B C L$ cell lines, including different $B C L-2$ rearranged and $\mathrm{DH}$ lymphoma models (Fig. $4 \mathrm{C}$ and $\mathrm{D}$ ). The observation that Prexasertib-induced apoptosis was less pronounced in $B C L-2$-rearranged and $\mathrm{DH}$ cell lines (Figure $S 5 \mathrm{C}, \mathrm{D}$ ) further corroborates these findings. $\mathrm{BCL}-2$ protein expression data in this validation panel are shown in supplement (Figure S8A). Although the efficacy of Prexasertib in combination with Venetoclax in DE cell lines was not affected by baseline $\gamma \mathrm{H} 2 \mathrm{AX}$ 
A

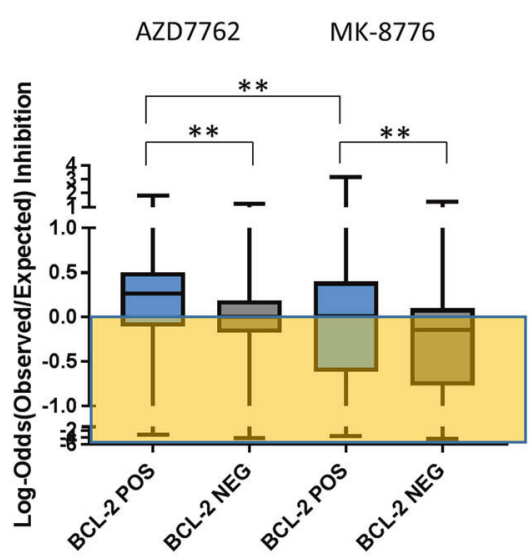

C

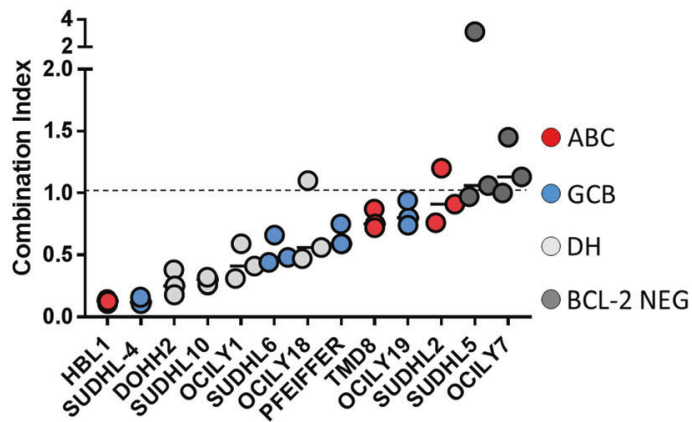

B

D
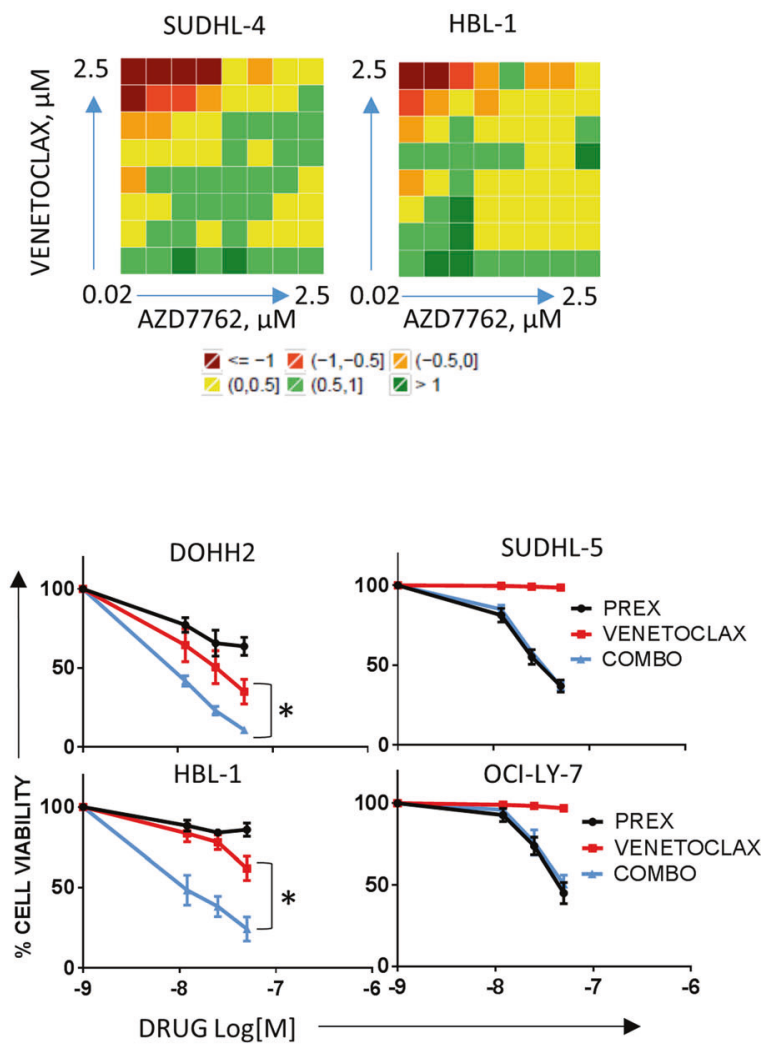

$E$
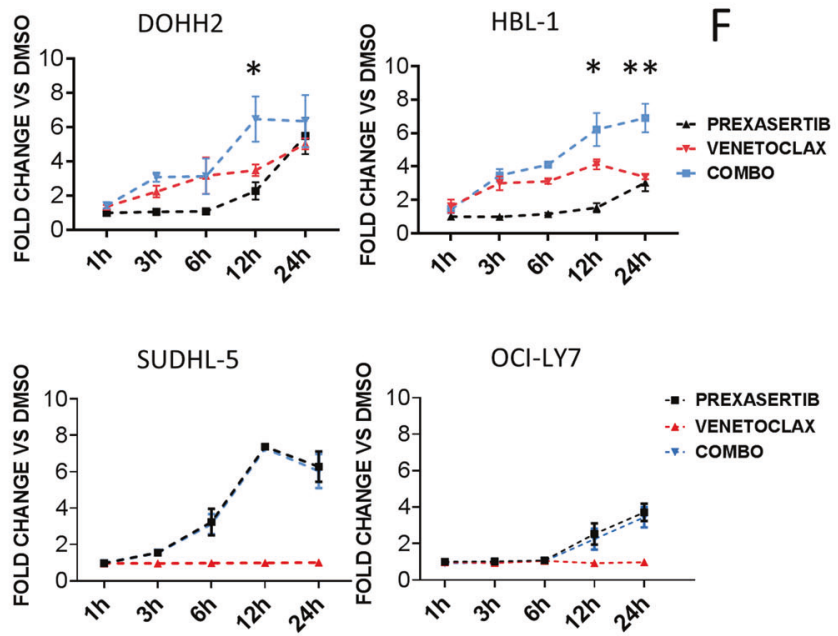

- PREXASERTIB

-. VENETOCLAX

.. Сомво

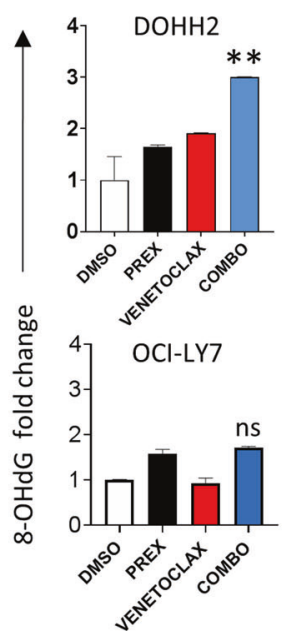

and 8-OHdG levels, cell lines with low levels of $\mathrm{\gamma H} 2 \mathrm{AX}$ and 8-OHdG showed a trend toward a decreased sensitivity to single-agent Venetoclax (Figure S8B-G). Combinatory treatment with Prexasertib and Venetoclax synergistically induced apoptosis as demonstrated by time-dependent increase in caspase 3/7 cleavage in $B C L-2$ positive $A B C$ and $D H$ lymphoma cell lines (Fig. $4 E$ ). In line with the data shown in Fig. 2 and with the role of BCL-2 in regulating the oxidative stress response, these changes were associated with early induction of oxidative DNA damage (Fig. 4F). Taken together these data indicate that selective BCL-2 blockade enhances the in vitro activity of $\mathrm{CHK}$ inhibitors in multiple $\mathrm{BCL}-2$ positive $\mathrm{DLBCL}$ models including $\mathrm{HG}-\mathrm{BCL} \mathrm{w} / \mathrm{DH}$, by inducing oxidative DNA damage and apoptosis. Of note, single-agent Prexasertib or oxidative stress-inducing agents such as doxorubicin or $\mathrm{H} 2 \mathrm{O} 2$ did not determine significant $\mathrm{BCL}-2$ induction in either BCL-2 negative or BCL-2 positive cell lines (Figure S9A). Finally, the MCL-1 inhibitor 568345 enhanced Prexasertib efficacy in $\mathrm{BCL}-2$ negative cell lines (Figure $\mathrm{S} 9 \mathrm{~B}$ ), suggesting that dual blockade of the DDR and alternative BCL-2 family members could be of value in DLBCLs with low BCL-2 expression. 
Fig. 4 BCL-2 blockade enhances antilymphoma activity of DDR inhibitors in vitro. A High throughput screening of the checkpoint kinase inhibitors AZD7762 or MK-8776 combined with the BCL-2 inhibitor Venetoclax (ABT-199) in 10 DLBCL cell lines. Box plot graphs summarize the results of the combinatorial drug screening analyzed with the Bliss model in $10 \mathrm{DLBCL}$ cell lines treated for $72 \mathrm{~h}$ with the indicated drug combinations. The $Y$ axis indicates the ratio between observed and expected inhibition in a log scale. Values above the " 0 " line indicate enhanced antiproliferative effects for the combinations. See HTS statistical analysis in the methods section and supplemental data for detailed information. See also Figure S6. B Representative examples of combination experiments of AZD7762 plus Venetoclax in HBL-1 and SUDHL-4 cells. Combination responses are examined using a $8 \times 8$ viability matrix representing the ratio between observed/expected inhibition in log scale, which was measured after $72 \mathrm{~h}$ post treatment. Full data are shown in supplement. $\mathrm{C}$ High throughput screening results were confirmed by independent experiments with the CHK1/2 inhibitor Prexasertib and Venetoclax, using the Cell Titer Glo assay in 13 DLBCL cell lines, and results were calculated using Combination Index analysis. Three doses of Prexasertib $(12.5,25,50 \mathrm{nM})$ were combined with three doses of Venetoclax $(12.5,25,50 \mathrm{nM})$ for $24 \mathrm{~h}$, allowing three combinatory values for each cell line. Values below 1 indicate synergistic interactions. ABC cell lines overexpressing BCL-2 are depicted in red, GCB in blue, DH in light gray, BCL-2 negative in dark gray. See also Figures S7 and S8. D Representative examples of Cell Titer Glo experiments in two BCL-2 positive (DOHH2, HBL-1), and 2 BCL-2 negative (OCILY-7, SUDHL-5) cell lines. Cells were incubated with increasing concentrations of Prexasertib (PREX) and Venetoclax (12.5, 25, 50 nM), and cell viability was assessed after $24 \mathrm{~h}$. Error bars represent standard error of the mean (S.E.M) of triplicate experiments. Differences between groups were calculated with the Student $T$ test. ${ }^{*} p<0.05,{ }^{* *} p<0.01$. See also Figures S7, S8 and S9. E Graph showing fold changes over time in caspase 3/7 activity in BCL-2 positive (DOHH2, HBL-1) vs BCL-2 negative (OCILY-7, SUDHL-5) cell lines treated with Prexasertib, Venetoclax, or the combination. Cells were treated with $50 \mathrm{nM}$ of Prexasertib, Venetoclax and the combination for the indicated time. Error bars represent standard error of the mean (S.E.M) of triplicate experiments. Differences between groups (combo vs Venetoclax and Prexasertib as single agents) were calculated with the Student $T$ test. ${ }^{*} p<0.05,{ }^{* *} p<0.01$. F Bar graph showing early changes in DNA oxidation levels in DOHH2 and OCILY-7 cells treated with Prexasertib, Venetoclax or the combination for $1 \mathrm{~h}$. After treatment, DNA was extracted and 8-OHdG levels were assessed with a dedicated ELISA assay. The combination determined synergistic induction of 8-OHdG in the BCL-2 positive DOHH2 cells and was ineffective in the BCL-2 negative cell line OCILY-7. Error bars represent standard error of the mean (S.E.M) of triplicate experiments. Differences between groups were calculated with the Student $T$ test. ${ }^{*} p<0.05,{ }^{* *} p<0.01$.

\section{Differential role of $M Y C$ and $B C L-2$ in modulating the efficacy of checkpoint kinase inhibition}

To determine the effects of $\mathrm{BCL}-2$ overexpression on the therapeutic efficacy of DDR inhibition, we generated a Tet-on inducible system to overexpress BCL-2 in the Prexasertib-sensitive BCL-2 negative cell line SUDHL-5. Cells were preincubated with doxycycline $1 \mu \mathrm{g} / \mathrm{ml}$ for $24 \mathrm{~h}$ to overexpress BCL-2, and then were treated with DMSO or Prexasertib for $24 \mathrm{~h}$ (Figure S10A). BCL-2 overexpression, while not exerting significant effects on cell proliferation in untreated cells, significantly decreased the efficacy of Prexasertib in this system, indicating that BCL-2 promotes resistance to DDR inhibition (Fig. 5A and S10A). These changes were associated with an attenuated induction of $\mathrm{YH} 2 \mathrm{AX}$ and with a decreased caspase 3 cleavage in cells overexpressing $B C L-2$ (Fig. 5B,C and S10A,B). BCL-2 overexpression did not determine significant changes in cell cycle dynamics under Prexasertib treatment (Fig. 5D).

To assess the role of MYC in regulating the antiproliferative activity of Prexasertib, we used the P-4936 cell line, which carries a conditional, tetracycline-regulated (Tet-OFF) MYC promoter $[35,36]$. Cells were pre-treated with DMSO or with doxycycline for $6 \mathrm{~h}$ to abrogate MYC expression, and then incubated with Prexasertib for $24 \mathrm{~h}$ (Fig. 5E-H and S11A-C). MYC silencing with doxycycline significantly reduced cell proliferation and baseline $\mathrm{\gamma H} 2 \mathrm{AX}$ expression (Figure S11A-C). Importantly MYC depletion reduced the antiproliferative effects of Prexasertib in these cells, which was associated with impaired $\mathrm{YH} 2 \mathrm{AX}$ induction, decreased caspase 3 cleavage, and attenuated effects on cell cycle dynamics (Fig. $5 \mathrm{E}-\mathrm{H}$ ). These data suggest that MYC and BCL-2 may modulate the sensitivity to CHK1 inhibition in opposite ways: in fact, while high MYC expression could be associated with increased DDR activation and enhanced susceptibility to DDRi-induced DNA damage, overexpression of BCL-2 may significantly decrease the therapeutic activity of DDR inhibitors, providing mechanistic rationale of dual blockade of $\mathrm{DDR}$ and $\mathrm{BCL}-2$ in $\mathrm{DE}$ and $\mathrm{DH}$ lymphomas.

\section{BCL-2 inhibition enhances the activity of DDR inhibitors} in vivo in DH PDX models

To assess whether our in vitro results could be confirmed in in vivo lymphoma models, we used a PDX mouse model harboring a double MYC and BCL-2 rearrangement and a $17 p$ deletion (TP53 loss) [42]. Treatment with Prexasertib as single-agent significantly extended the survival of mice bearing DH lymphomas (Fig. 6A). Of note, a short course 3-week therapy schedule was sufficient to extend survival of several weeks in this mouse model. In order to assess the in vivo effects of CHK inhibition, lysates from bone marrow and spleens harvested after $6 \mathrm{~h}$ of vehicle or Prexasertib administration were subjected to western blotting. In line with our in vitro data, Prexasertib treatment resulted in increased $\mathrm{YH} 2 \mathrm{AX}$ and p-CHK1 s345 levels, which are established biomarkers of DDR inhibition, indicative of DNA damage accumulation and ATRdependent $\mathrm{CHK} 1$ phosphorylation (Fig. 6B). In an independent experiment, we investigated the efficacy of Prexasertib in combination with the BCL-2 inhibitor Venetoclax. In vivo combination therapy with Prexasertib and Venetoclax exerted synergistic effects in our DH PDX model. Prexasertib as singleagent confirmed high antitumor activity resulting in extended survival. On the contrary, Venetoclax alone had no substantial antitumor activity. However, the combination of Prexasertib and Venetoclax resulted in enhanced tumor growth inhibition and prolonged survival, as compared to either drug administered as single agent (Fig. 6C-E). Interestingly these synergistic effects were observed after only one cycle ( 3 weeks) of combined treatment. We did not observe significant weight loss in mice treated with Prexasertib, Venetoclax, or the combination (Figure S12 A and B). Collectively these data suggest that combined DDR and BCL-2 inhibition could be an effective treatment strategy in $\mathrm{DH}$ lymphoma models, including those with defective p53 axis.

\section{DISCUSSION}

In an effort to understand the functional basis of the intrinsic chemoresistance associated with increased MYC and BCL-2 levels in a significant fraction of DLBCL, we hypothesized that: 1) Overexpression of DDR and oxidative DNA damage markers $(\mathrm{\gamma H} 2 \mathrm{AX}$ and $8-\mathrm{OHdG})$ could identify poor prognosis DLBCL subsets. 2) Pharmacologic inhibition of the DDR and antioxidant response through combined $\mathrm{CHK} 1 / 2$ and $\mathrm{BCL}-2$ blockade could unleash endogenous replicative and oxidative stress resulting in synergistic therapeutic activity in MYC/BCL-2 positive DLBCL.

To address these hypotheses, we first profiled two independent DLBCL cohorts with T-GEP, FISH, and IHC, in order to define COO subtyping, MYC, and BCL-2 status and expression levels of 
A

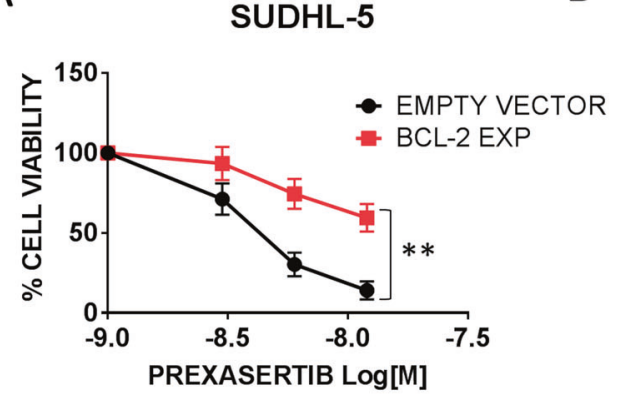

C

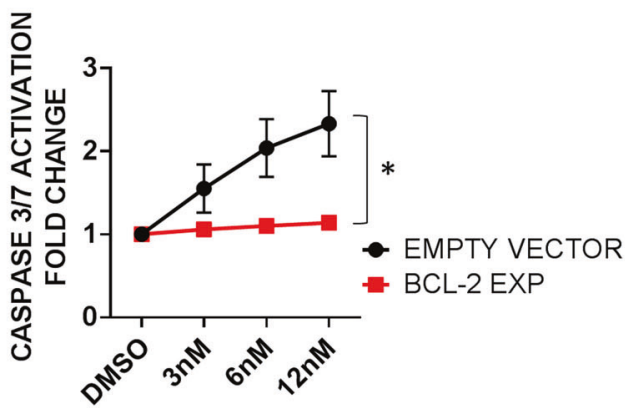

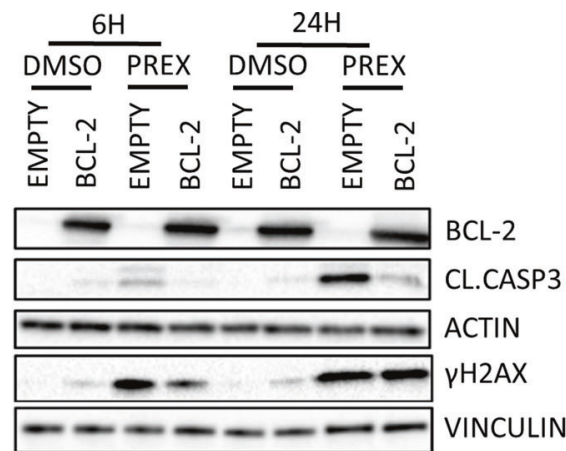

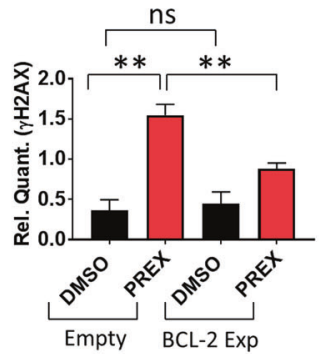

D

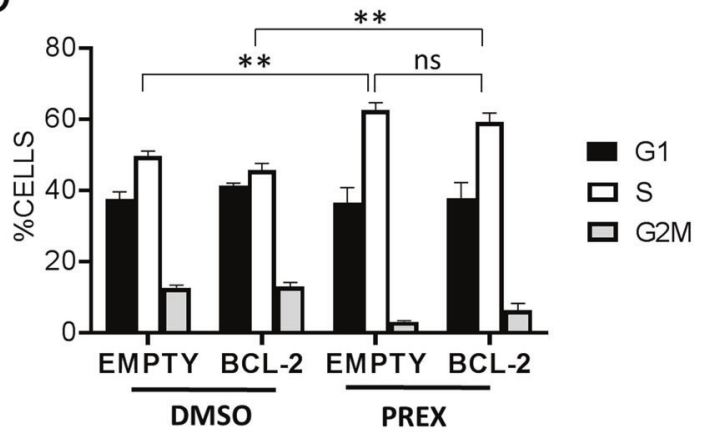

E

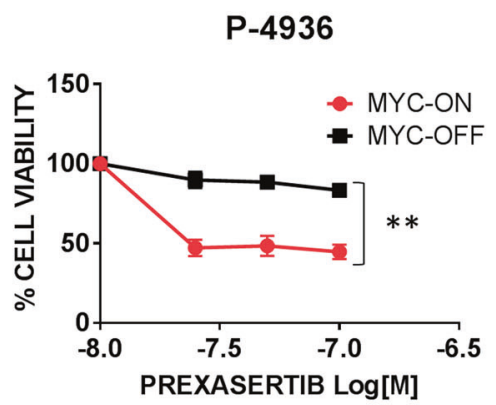

G

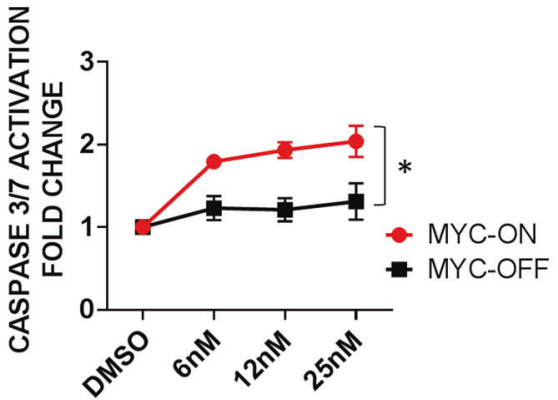

$\mathrm{F}$
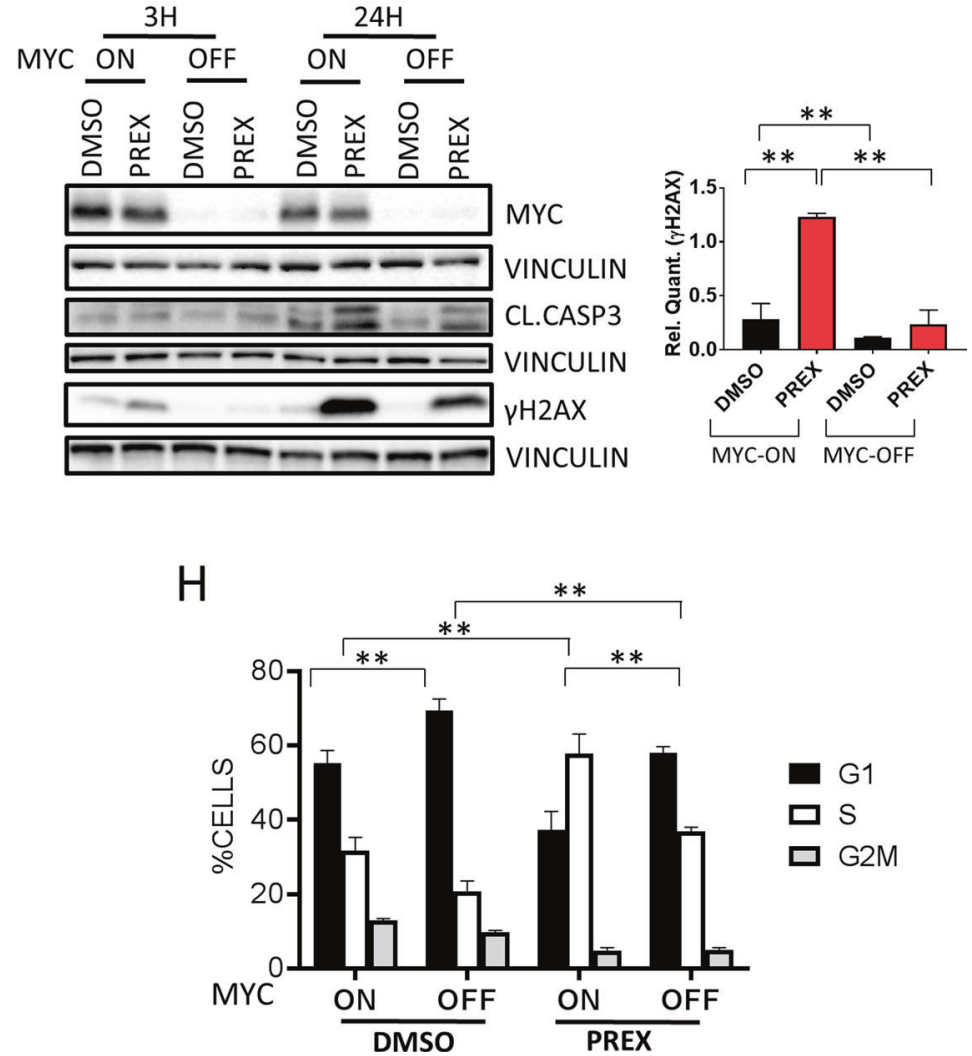

biomarkers of DDR activation and oxidative DNA damage $(\mathrm{\gamma H} 2 \mathrm{AX}$ and $8-\mathrm{OHdG}$ ). We demonstrated that: 1) expression levels of the DNA damage marker $\mathrm{\gamma H} 2 \mathrm{AX}$ and the oxidative DNA damage marker $8-\mathrm{OHdG}$ are tightly associated, suggesting that oxidative

stress could be a major source of inherent DNA damage contributing to constitutive DDR activation in DLBCL (Fig. 2); 2) Dual positivity for $\mathrm{YH} 2 \mathrm{AX}$ and $8-\mathrm{OHdG}$ is significantly associated with adverse outcome in the MYC/BCL-2 positive DLBCL subset 
Fig. 5 Role of BCL-2 and MYC in modulating sensitivity to DDR inhibition. A Cell Titer Glo assay showing the effects of 3 doses of Prexasertib $(3,6$, and $12 \mathrm{nM}$ ) for $24 \mathrm{~h}$ in the absence (EMPTY VECTOR) or in the presence of BCL-2 (BCL-2 EXP). SUDHL-5 cells (transfected with EMPTY VECTOR or a BCL-2 TET-ON inducible system (BCL-2 EXP) were preincubated with doxycycline $1 \mu \mathrm{g} / \mathrm{ml}$ for $24 \mathrm{~h}$ and then treated with Prexasertib at the indicated doses (see also Figure S10A). Error bars represent standard error of the mean (S.E.M) of triplicate experiments. Differences between groups were calculated with the Student $T$ test. ${ }^{*} p<0.05,{ }^{* *} p<0.01$. B On the left, representative western blot assay showing the effects of Prexasertib (6 nM) (PREX) on $\gamma \mathrm{H} 2 \mathrm{AX}$ induction and caspase 3 cleavage (CL. CASP 3 ) in the presence or absence of BCL-2, after 6 and $24 \mathrm{~h}$ of incubation. On the right, quantitative densitometry analyses (ImageJ software, western blots are shown in Figure S10B) showing normalized $\gamma \mathrm{H} 2 \mathrm{AX}$ levels vs vinculin after $6 \mathrm{~h}$ of incubation with Prexasertib in the presence or absence of $\mathrm{BCL}-2$ : $\gamma \mathrm{H} 2 \mathrm{AX}$ induction by Prexasertib was significantly attenuated in the presence of BCL-2. Error bars represent standard error of the mean (S.E.M) of triplicate experiments. Differences between groups were calculated with the Student $T$ test. ${ }^{*} p<0.05,{ }^{* *} p<0.01$. C Graph showing fold changes in caspase 3/7 activity in SUDHL-5 cells treated with increasing doses of Prexasertib $(3,6,12 \mathrm{nM})$ for $12 \mathrm{~h}$ in the presence or absence of BCL-2 (BCL-2 EXP vs EMPTY VECTOR), showing significant inhibition of caspase 3/7 cleavage in BCL-2 overexpressing SUDHL-5 cells. Error bars represent standard error of the mean (S.E.M) of triplicate experiments. Differences between groups were calculated with the Student $T$ test. ${ }^{*} p$ $<0.05,{ }^{* *} p<0.01$. D Bar graph showing the effects of DMSO or Prexasertib (PREX) on cell cycle phases in SUDHL-5 cells in the presence or absence of BCL-2. After overexpression of BCL-2 (BCL-2) or the empty vector (EMPTY) for $24 \mathrm{~h}$, cells were incubated with $12 \mathrm{nM}$ Prexasertib for additional $24 \mathrm{~h}$ and cell cycle phases assessed by flow cytometry (propidium iodide staining). Error bars represent standard error of the mean (S.E.M) of triplicate experiments. Differences between groups were calculated with the Student $T$ test. ${ }^{*} p<0.05,{ }^{* *} p<0.01$. E Cell Titer Glo assay showing the effects of three doses of Prexasertib $(25,50$, and $100 \mathrm{nM})$ for $24 \mathrm{~h}$ in the absence of MYC (MYC OFF) or in the presence of MYC (MYC-ON) in P-4936 cells. P-4936 cells (carrying a tetracycline inducible promoter, TET-OFF MYC) were preincubated with doxycycline 1 $\mu \mathrm{g} / \mathrm{ml}$ for $6 \mathrm{~h}$ to downregulate MYC, and then treated with Prexasertib at the indicated doses (See also Figure S11A). Error bars represent standard error of the mean (S.E.M) of triplicate experiments. Differences between groups were calculated with the Student $T$ test. ${ }^{*} p<0.05$, ${ }^{* *} p<0.01$. F Representative western blot assay showing the effects of Prexasertib ( $25 \mathrm{nM}$ for 3 and $24 \mathrm{~h}$ of incubation) (PREX) on $\gamma \mathrm{H} 2 \mathrm{AX}$ induction and caspase 3 cleavage in the presence (MYC-ON) or absence of MYC (MYC-OFF). On the right, quantitative densitometry analyses (ImageJ software, western blots are shown in Figure S11B) showing normalized $\gamma \mathrm{H} 2 \mathrm{AX}$ levels vs vinculin after $3 \mathrm{~h}$ of incubation with Prexasertib in MYC-ON and MYC-OFF P-4936 cells. $\gamma \mathrm{H} 2 \mathrm{AX}$ induction by Prexasertib was significantly attenuated in the absence of MYC. Error bars represent standard error of the mean (S.E.M) of triplicate experiments. Differences between groups were calculated with the Student $T$ test. ${ }^{*} p<0.05,{ }^{* *} p<0.01$. G Graph showing fold changes in caspase $3 / 7$ activity in P-4936 cells treated with increasing doses of Prexasertib (6, $12,25 \mathrm{nM}$ ) for $12 \mathrm{~h}$ in the presence or absence of MYC (MYC-ON vs MYC-OFF), showing significant inhibition of caspase 3/7 cleavage in MYCOFF P-4936 cells. Error bars represent standard error of the mean (S.E.M) of triplicate experiments. Differences between groups were calculated with the Student $T$ test. ${ }^{*} p<0.05,{ }^{* *} p<0.01$. H Bar graph showing the effects of DMSO or Prexasertib (PREX) on cell cycle phases in P-4936 cells in the presence or absence of MYC. After preincubation with doxycycline (MYC-OFF) or DMSO (MYC-ON) for $24 \mathrm{~h}$, cells were incubated with $25 \mathrm{nM}$ Prexasertib for additional $24 \mathrm{~h}$ and cell cycle phases assessed by flow cytometry (propidium iodide staining). Error bars represent standard error of the mean (S.E.M) of triplicate experiments. Differences between groups were calculated with the Student $T$ test. ${ }^{*} p$ $<0.05,{ }^{* *} p<0.01$.

(Fig. 2); 3) $M Y C / B C L-2$ mRNA DE DLBCL overexpressing $\mathrm{YH} 2 \mathrm{AX}$ and 8-OHdG (DE-OX high) are enriched of $A B C$ and $D H$ cases, and are characterized by increased $B C L-2$ mRNA expression compared to their $\mathrm{YH} 2 \mathrm{AX}$ and $8-\mathrm{OHdG}$ negative DE counterparts. Importantly all but one HG-BCL w/DH cases clustered in the DE-OX high subgroup (Fig. 2, Table 2). These data indicate that a subgroup of $A B C D L B C L$ and $H G-B C L$ w/DH are characterized by high levels of inherent oxidative DNA damage, these features being associated with increased $B C L-2$ expression levels. Given the poor prognosis of these DLBCL subsets, the well-established oncogenic cooperation between MYC and BCL-2, and the known role of BCL2 in oxidative stress response, these data are in line with a model whereby $\mathrm{BCL}-2$ overexpression and constitutive DDR activation could provide a tolerance mechanism to MYC-induced replicative and oxidative stress. Since antracyclines exert their cytotoxic activity at least in part by increasing ROS levels $[52,53]$ and determining oxidative DNA damage, lymphoma subsets displaying inherent tolerance to oxidative DNA damage through constitutive DDR activation and BCL-2 overexpression could be intrinsically resistant to current antracycline-based chemotherapeutic regimens. The results of our in vitro experiments support this hypothesis since DDR inhibition by Prexasertib determined oxidative DNA damage accumulation (Fig. 3), which was further enhanced by the addition of Venetoclax (Fig. 4). Notably, treatment with ROS-inducing agents (Antimycin A) and Prexasertib determined accumulation of oxidative DNA damage to similar extents, suggesting that constitutive DDR activation could have a major role in preventing intolerable levels of DNA damage and genomic instability induced by endogenous oxidative stress (Fig. 3). BCL-2 blockade with Venetoclax enhanced the antilymphoma activity of checkpoint kinase inhibitors in multiple BCL-2 positive cell lines (including $A B C$ and DH DLBCL models) resulting in increased apoptosis (Fig. 4). While enforced BCL-2 expression significantly decreased the efficacy of single-agent DDR inhibition by attenuating DNA damage accumulation and apoptosis induction (Fig. 5), on the contrary, MYC overexpression was associated with increased sensitivity to DDR inhibitors, and enhanced apoptotic response in line with previous reports [21-23]. Interestingly, while BCL-2 overexpression did not exert significant effects on cell proliferation, ectopic MYC expression was associated with increased cell proliferation and enhanced $\mathrm{yH} 2 \mathrm{AX}$ expression, indicative of increased replicative stress and DDR activation (Figure S11). These observations underline the intrinsic correlation between MYC and the DDR, and the importance of dual targeting of the DDR and $B C L-2$ in $M Y C / B C L-2$ positive lymphoma in order to maximize the therapeutic efficacy. These data were confirmed in vivo, in a double hit PDX model with TP53 loss (Fig. 6). Interestingly single-agent Venetoclax had negligible antilymphoma activity in vivo in line with data from early phase clinical trials in DLBCL [54]. The recent demonstration of synergy between Venetoclax and Tygecycline in $\mathrm{DH}$ lymphoma models is in line with our findings, indicating that therapeutic strategies based on synthetic lethal targeting of oxidative stress could be of value in DH-DLBCL [55].

In summary, these data indicate that increased tolerance to replicative and oxidative stress through DDR activation and BCL-2 overexpression could be a unifying feature of poor prognosis MYC positive DLBCL subsets such as $A B C$ and $H G-B C L$ w/DH, which could be the basis for a tailored therapeutic approach. In this light, novel therapies based on dual targeting of DDR and antioxidant response could determine significant improvements in DLBCL therapy. This strategy, based on unleashing endogenous MYCrelated replicative and oxidative stress rather than inducing exogenous DNA damage, represents a significant innovation, which could provide less toxic alternatives to conventional chemotherapy. 
A

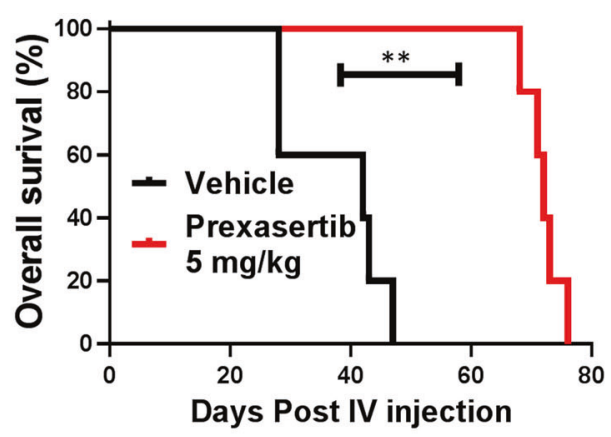

C

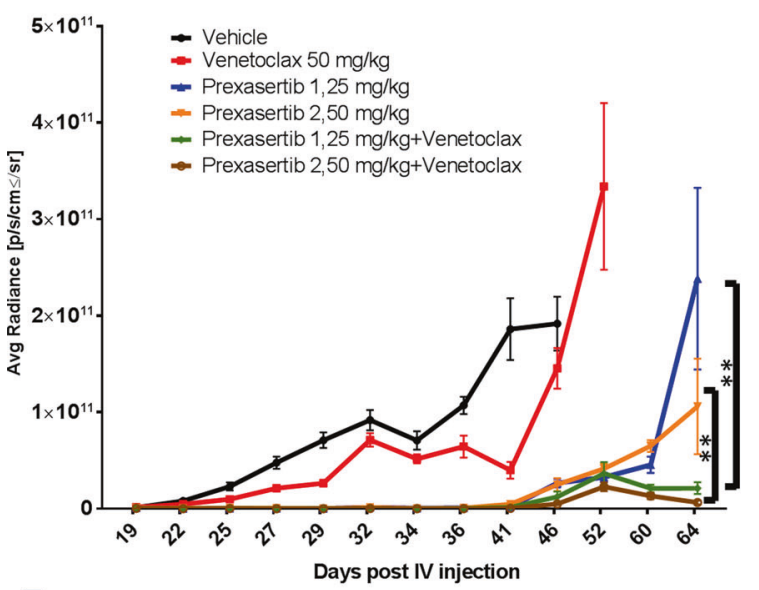

E

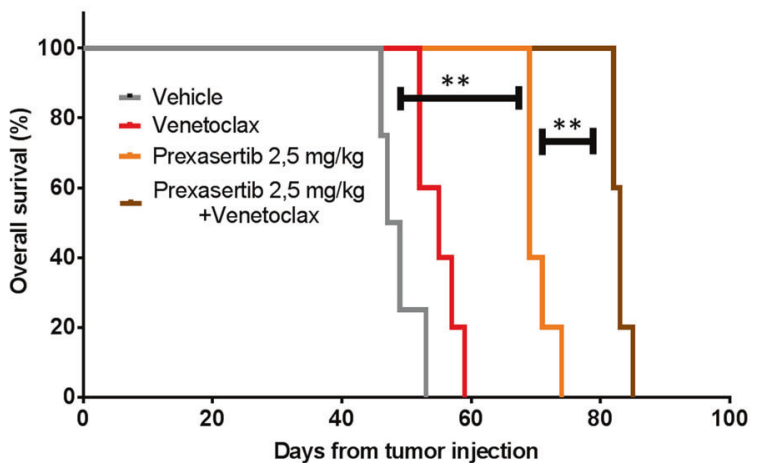

D
B
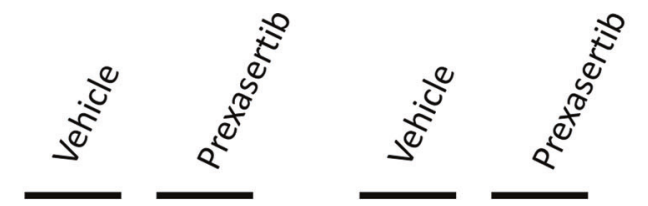

Lamin B1

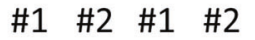

pCHK1 S345

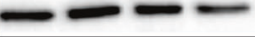

$\gamma \mathrm{H} 2 \mathrm{AX}$

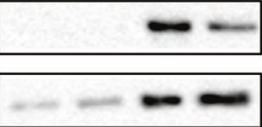

Bone Marrow

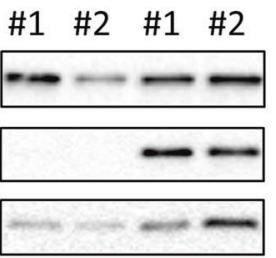

Spleen

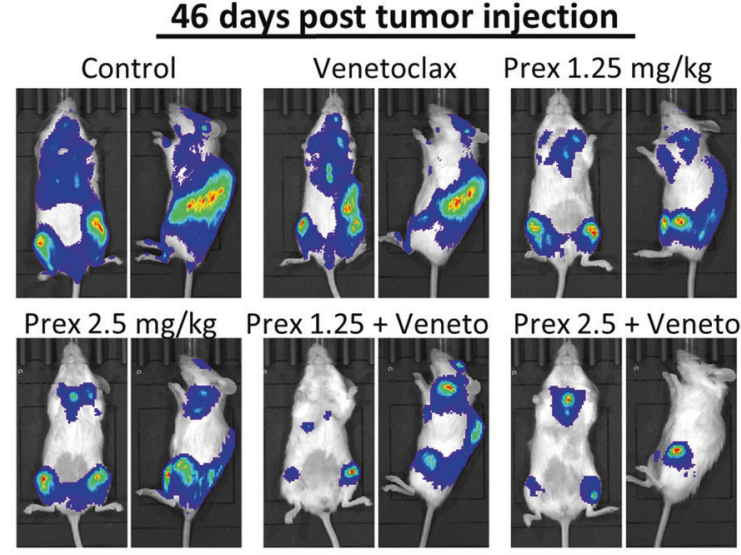

64 days post tumor injection

Prex $1.25 \mathrm{mg} / \mathrm{kg}$ Prex $2.5 \mathrm{mg} / \mathrm{kg}$
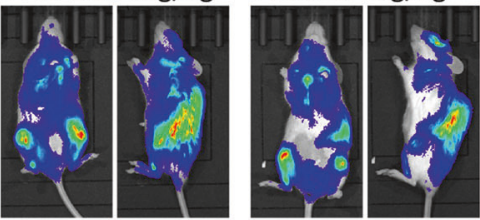

Prex $1.25+$ Veneto

Prex $2.5+$ Veneto

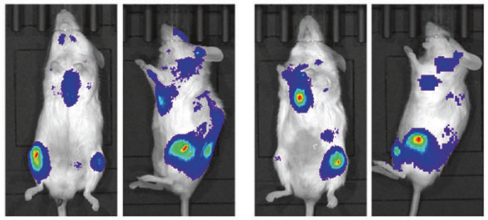

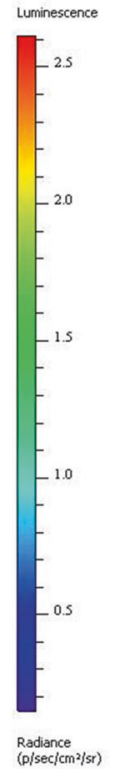


Fig. 6 Prexasertib plus Venetoclax extends survival in vivo in a PDX DH lymphoma model. A Overall survival curve of PDX mice treated with vehicle $(n=5)$ or two different doses of Prexasertib ( 5 and $10 \mathrm{mg} / \mathrm{kg}$ BID 3 times/week) $\left(n=5\right.$ in each dose level). Cells $\left(10^{6}\right)$ were xenografted via tail vein injection into six- to eight-week-old female NSG mice (Charles River, Italy). Tumor growth was monitored three times per week by whole-body imaging on an IVIS Lumina III platform. Mice were treated for three weeks (nine doses). $P$ values were calculated with the log rank test. B Western blot showing the in vivo effects of Prexasertib (5 mg/kg BID three times/week) on $\gamma \mathrm{H} 2 \mathrm{AX}$ and p-CHK1-S345 levels. Mice were sacrificed at the end of treatment $(6 \mathrm{~h}$ after last dosing), and tissues were harvested immediately after sacrifice. C Combination experiment of Prexasertib and Venetoclax in the DH lymphoma PDX model (DFBL-69487-V3-mCLP). NSG mice were treated with vehicle $(n=$ 5), Prexasertib ( 1.25 and $2.5 \mathrm{mg} / \mathrm{kg}$ BID three times/week) ( $n=5$ in each dose level), Venetoclax ( $50 \mathrm{mg} / \mathrm{Kg} / \mathrm{daily}$ (five days/week) by oral gavage $(n=5)$ and the combinations ( $n=5$ each). Mice were treated for three weeks. Differences between groups were calculated with the Student $T$ test. ${ }^{*} p<0.05,{ }^{* *} p<0.01$. D Representative IVIS imaging of mice treated with vehicle (control), Venetoclax, Prexasertib (Prex) 1.25 $\mathrm{mg} / \mathrm{kg}$, Prexasertib $2.5 \mathrm{mg} / \mathrm{kg}$, and the combinations at the indicated time points. All vehicle and Venetoclax-treated mice were sacrificed before day 64. E Overall survival curve of PDX mice treated with vehicle, two different doses of Prexasertib (1.25 and $2.5 \mathrm{mg} / \mathrm{kg}$ three times/ week) ( $n=5$ in each dose level), Venetoclax $50 \mathrm{mg} / \mathrm{Kg}$ (five days/ week) $(n=5)$ and the combinations $(n=5$ each). Mice were treated for a total of 21 days. $P$ values were calculated with the log rank test. See also Figure S12.

\section{REFERENCES}

1. Coiffier B, Lepage E, Briere J, Herbrecht R, Tilly H, Bouabdallah R, et al. CHOP chemotherapy plus rituximab compared with $\mathrm{CHOP}$ alone in elderly patients with diffuse large-B-cell lymphoma. N. Engl J Med. 2002;346:235-42.

2. Sehn LH, Donaldson J, Chhanabhai M, Fitzgerald C, Gill K, Klasa R, et al. Introduction of combined CHOP plus rituximab therapy dramatically improved outcome of diffuse large B-cell lymphoma in British Columbia. J Clin Oncol. 2005;23:5027-33.

3. Ziepert M, Hasenclever D, Kuhnt E, Glass B, Schmitz N, Pfreundschuh $M$, et al. Standard International prognostic index remains a valid predictor of outcome for patients with aggressive CD20+ B-cell lymphoma in the rituximab era. J Clin Oncol. 2010;28:2373-80.

4. Alizadeh AA, Eisen MB, Davis RE, Ma C, Lossos IS, Rosenwald A, et al. Distinct types of diffuse large B-cell lymphoma identified by gene expression profiling. Nature 2000;403:503-11.

5. Shipp MA, Ross KN, Tamayo P, Weng AP, Kutok JL, Aguiar RC, et al. Diffuse large B-cell lymphoma outcome prediction by gene-expression profiling and supervised machine learning. Nat Med. 2002;8:68-74.

6. Scott DW, Wright GW, Williams PM, Lih CJ, Walsh W, Jaffe ES, et al. Determining cell-of-origin subtypes of diffuse large B-cell lymphoma using gene expression in formalin-fixed paraffin-embedded tissue. Blood 2014;123:1214-7.

7. Scott DW, Mottok A, Ennishi D, Wright GW, Farinha P, Ben-Neriah S, et al. Prognostic significance of diffuse large B-cell lymphoma cell of origin determined by digital gene expression in formalin-fixed paraffin-embedded tissue biopsies. J Clin Oncol. 2015;33:2848-56.

8. Hu S, Xu-Monette ZY, Tzankov A, Green T, Wu L, Balasubramanyam A, et al. MYC/ $\mathrm{BCL} 2$ protein coexpression contributes to the inferior survival of activated B-cell subtype of diffuse large B-cell lymphoma and demonstrates high-risk gene expression signatures: a report from The International DLBCL Rituximab-CHOP Consortium Program. Blood 2013;121:4021-31.

9. Davis RE, Brown KD, Siebenlist U, Staudt LM. Constitutive nuclear factor kappaB activity is required for survival of activated B cell-like diffuse large B cell lymphoma cells. J Exp Med. 2001;194:1861-74.

10. Younes A, Sehn LH, Johnson P, Zinzani PL, Hong X, Zhu J, et al. Randomized phase III trial of ibrutinib and rituximab plus cyclophosphamide, doxorubicin, vincristine, and prednisone in non-germinal center b-cell diffuse large B-cell lymphoma. J Clin Oncol. 2019;37:1285-95.

11. Vitolo U, Witzig T, Gascoyne R, Scott DW, Zhang Q, Jurczak W, et al. ROBUST: first report of phase III randomized study of lenalidomide/R-CHOP $\left(\mathrm{R}^{2}-\mathrm{CHOP}\right)$ vs placebo/R-CHOP in previously untreated ABC-type diffuse large B-cell lymphoma. Hematol Oncol. 2019;37:36-37.

12. Davies A, Cummin TE, Barrans S, Maishman T, Mamot C, Novak U, et al. Geneexpression profiling of bortezomib added to standard chemoimmunotherapy for diffuse large B-cell lymphoma (REMoDL-B): an open-label, randomised, phase 3 trial. Lancet Oncol. 2019;20:649-62.

13. Green TM, Young KH, Visco C, Xu-Monette ZY, Orazi A, Go RS, et al. Immunohistochemical double-hit score is a strong predictor of outcome in patients with diffuse large B-cell lymphoma treated with rituximab plus cyclophosphamide, doxorubicin, vincristine, and prednisone. J Clin Oncol. 2012;30:3460-7.

14. Johnson NA, Slack GW, Savage KJ, Connors JM, Ben-Neriah S, Rogic S, et al. Concurrent expression of MYC and BCL2 in diffuse large B-cell lymphoma treated with rituximab plus cyclophosphamide, doxorubicin, vincristine, and prednisone. J Clin Oncol. 2012;30:3452-9.

15. Swerdlow SH, Campo E, Harris NL, Jaffe ES, Pileri SA, Stein H et al. WHO classification of tumours of the haematopoietic and lymphoid tissues. Revised 4th ed. Lyon, France: IARC; 2017.

16. Friedberg JW. How I treat double-hit lymphoma. Blood 2017;130:590-6.
17. Petrich AM, Gandhi M, Jovanovic B, Castillo JJ, Rajguru S, Yang DT, et al. Impact of induction regimen and stem cell transplantation on outcomes in double-hit lymphoma: a multicenter retrospective analysis. Blood 2014;124:2354-61.

18. Halazonetis TD, Gorgoulis VG, Bartek J. An oncogene-induced DNA damage model for cancer development. Science 2008;319:1352-5.

19. Negrini S, Gorgoulis VG, Halazonetis TD. Genomic instability-an evolving hallmark of cancer. Nat Rev Mol Cell Biol. 2010;11:220-8.

20. Vafa O, Wade M, Kern S, Beeche M, Pandita TK, Hampton GM, et al. c-Myc can induce DNA damage, increase reactive oxygen species, and mitigate p53 function: a mechanism for oncogene-induced genetic instability. Mol Cell. 2002;9:1031-44.

21. Rohban S, Campaner S. Myc induced replicative stress response: how to cope with it and exploit it. Biochim Biophys Acta. 2015;1849:517-24.

22. Kuzyk A, Mai S. c-MYC-induced genomic instability. Cold Spring Harb Perspect Med. 2014;4:a014373.

23. Murga M, Campaner S, Lopez-Contreras AJ, Toledo LI, Soria R, Montaña MF, et al. Exploiting oncogene-induced replicative stress for the selective killing of Mycdriven tumors. Nat Struct Mol Biol. 2011;18:1331-5.

24. Egler RA, Fernandes E, Rothermund K, Sereika S, de Souza-Pinto N, Jaruga $P$, et al. Regulation of reactive oxygen species, DNA damage, and c-Myc function by peroxiredoxin 1. Oncogene 2005;24:8038-50.

25. Graves JA, Metukuri M, Scott D, Rothermund K, Prochownik. EV. Regulation of reactive oxygen species homeostasis by peroxiredoxins and c-Myc. J Biol Chem 2009;284:6520-9.

26. Strasser A, Harris AW, Bath ML, Cory S. Novel primitive lymphoid tumours induced in transgenic mice by cooperation between myc and bcl-2. Nature 1990;348:331-3.

27. Fanidi A, Harrington EA, Evan Gl. Cooperative interaction between c-myc and bcl2 proto-oncogenes. Nature 1992;359:554-6.

28. Susnow N, Zeng L, Margineantu D, Hockenbery DM. Bcl-2 family proteins as regulators of oxidative stress. Semin Cancer Biol. 2009;19:42-9.

29. Derenzini E, Agostinelli C, Imbrogno E, lacobucci I, Casadei B, Brighenti E, et al. Constitutive activation of the DNA damage response pathway as a novel therapeutic target in diffuse large B-cell lymphoma. Oncotarget 2015;6:6553-69.

30. Xu-Monette ZY, Wu L, Visco C, Tai YC, Tzankov A, Liu WM, et al. Mutational profile and prognostic significance of TP53 in diffuse large B-cell lymphoma patients treated with R-CHOP: report from an International DLBCL Rituximab-CHOP Consortium Program Study. Blood 2012;120:3986-96.

31. Jardin F, Jais JP, Molina TJ, Parmentier F, Picquenot JM, Ruminy P, et al. Diffuse large $\mathrm{B}$-cell lymphomas with CDKN2A deletion have a distinct gene expression signature and a poor prognosis under R-CHOP treatment: a GELA study. Blood 2010;116:1092-104.

32. Derenzini E, Rossi A, Treré D. Treating hematological malignancies with drugs inhibiting ribosome biogenesis: when and why. J Hematol Oncol. 2018;11 (May):75.

33. Restelli V, Lupi M, Chilà R, Vagni M, Tarantelli C, Spriano F, et al. DNA damage response inhibitor combinations exert synergistic antitumor activity in aggressive B-cell lymphomas. Mol Cancer Ther. 2019;18:1255-64.

34. Chiappella A, Martelli M, Angelucci E, Brusamolino E, Evangelista A, Carella AM, et al. Rituximab-dose-dense chemotherapy with or without high-dose chemotherapy plus autologous stem-cell transplantation in high-risk diffuse large Bcell lymphoma (DLCL04): final results of a multicentre, open-label, randomised, controlled, phase 3 study. Lancet Oncol. 2017;18:1076-88.

35. Pajic A, Spitkovsky D, Christoph B, Kempkes B, Schuhmacher M, Staege MS, et al. Cell cycle activation by c-myc in a burkitt lymphoma model cell line. Int J Cancer. 2000;87:787-93. 
36. Zeller KI, Zhao X, Lee CW, Chiu KP, Yao F, Yustein JT, et al. Global mapping of cMyc binding sites and target gene networks in human B cells. Proc Natl Acad Sci USA. 2006;103:17834-9.

37. Derenzini E, Mondello P, Erazo T, Portelinha A, Liu Y, Scallion M, et al. BET inhibition-induced GSK3 $\beta$ feedback enhances lymphoma vulnerability to PI3K inhibitors. Cell Rep. 2018;24:2155-66.

38. Feller W. An introduction to probability theory and its applications. New York, NY: John Wiley \& Sons; 1971.

39. Tallarida RJ. Drug synergism: its detection and applications. J Pharm Exp Ther. 2001;298:865-72.

40. Kilkenny C, Browne WJ, Cuthill IC, Emerson M, Altman DG. Improving bioscience research reporting: the ARRIVE guidelines for reporting animal research. PLoS Biol. 2010;8:e1000412.

41. Townsend EC, Murakami MA, Christodoulou A, Christie AL, Koster J, DeSouza TA, et al. The public repository of xenografts enables discovery and randomized phase II-like trials in mice. Cancer Cell. 2016;29:574-86.

42. Kaplan EL, Meier P. Nonparametric estimations from incomplete observations. J Am Stat Assoc. 1958;53:457-81.

43. Chou TC. Drug combination studies and their synergy quantification using the Chou-Talalay method. Cancer Res. 2010;70:440-6.

44. R Core Team. R: a language and environment for statistical computing. R Foundation for Statistical Computing, Vienna, Austria. 2014. http://www.R-project.org/.

45. Kinner A, Wu W, Staudt C, lliakis G. Gamma-H2AX in recognition and signaling of DNA double-strand breaks in the context of chromatin. Nucleic Acids Res. 2008;36:5678-94.

46. Wu J, Clingen PH, Spanswick VJ, Mellinas-Gomez M, Meyer T, Puzanov I, et al. $\gamma$ $\mathrm{H} 2 \mathrm{AX}$ foci formation as a pharmacodynamic marker of DNA damage produced by DNA cross-linking agents: results from 2 phase I clinical trials of SJG-136 (SG2000). Clin Cancer Res. 2013;19:721-30.

47. Valavanidis A, Vlachogianni T, Fiotakis C. 8-hydroxy-2' -deoxyguanosine (8OHdG): a critical biomarker of oxidative stress and carcinogenesis. J Environ Sci Health C Environ Carcinog Ecotoxicol Rev. 2009;27:120-39.

48. King C, Diaz HB, McNeely S, Barnard D, Dempsey J, Blosser W, et al. LY2606368 causes replication catastrophe and antitumor effects through CHK1-dependent mechanisms. Mol Cancer Ther. 2015;14:2004-13.

49. Parmar K, Kochupurakkal BS, Lazaro JB, Wang ZC, Palakurthi S, Kirschmeier PT, et al. The CHK1 Inhibitor Prexasertib Exhibits Monotherapy Activity in High-Grade Serous Ovarian Cancer Models and Sensitizes to PARP Inhibition. Clin Cancer Res. 2019;25:6127-40.

50. Huang LS, Cobessi D, Tung EY, Berry EA. Binding of the respiratory chain inhibitor antimycin to the mitochondrial bc1 complex: a new crystal structure reveals an altered intramolecular hydrogenbonding pattern. J Mol Biol. 2005;351:573-97.

51. Ghelli Luserna Di Rorà A, lacobucci I, Imbrogno E, Papayannidis C, Derenzini E, Ferrari A, et al. Prexasertib, a Chk1/Chk2 inhibitor, increases the effectiveness of conventional therapy in B-/T- cell progenitor acute lymphoblastic leukemia. Oncotarget 2016;7:53377-91.

52. Mai Y, Yu JJ, Bartholdy B, Xu-Monette ZY, Knapp EE, Yuan F, et al. An oxidative stress-based mechanism of doxorubicin cytotoxicity suggests new therapeutic strategies in ABC-DLBCL. Blood 2016;128:2797-807.

53. Mizutani H, Tada-Oikawa S, Hiraku Y, Kojima M, Kawanishi S. Mechanism of apoptosis induced by doxorubicin through the generation of hydrogen peroxide. Life Sci. 2005;76:1439-53.

54. Davids MS, Roberts AW, Seymour JF, Pagel JM, Kahl BS, Wierda WG, et al. Phase I first-in-human study of Venetoclax in patients with relapsed or refractory nonhodgkin lymphoma. J Clin Oncol. 2017;35:826-33.

55. Ravà M, D'Andrea A, Nicoli P, Gritti I, Donati G, Doni M et al. Therapeutic synergy between tigecycline and venetoclax in a preclinical model of MYC/BCL2 doublehit B cell lymphoma. Sci Transl Med. 2018;10:eaan8723

\section{ACKNOWLEDGEMENTS}

This work was supported by Banca del Piemonte (Torino, Italy). AIRC (Italian Association for Cancer Research, Milan, Italy; $5 \times 1000$ grant n. 21198 to SP; AIRC IG20109 to FB), and the Italian Ministry of Health with Ricerca Corrente and 5×1000 funds. We would like to thank Dr. Anas Younes (MSKCC) for his helpful comments and discussion on data interpretation.

\section{AUTHOR CONTRIBUTIONS}

$E D$ and $A R$ conceived the study, performed experiments, analyzed the data and wrote the manuscript; SO and PF performed in vivo studies; AC, VT, SF, CA and SP performed, analyzed and reviewed immunohistochemistry studies; CC performed FISH studies, FB supervised in vivo studies; FM and GM performed T-GEP; SM analyzed T-GEP data; BC helped with data collection and data analysis; SP, FB, UV, PLZ, MA, PGP, CT helped with data interpretation and critically revised the manuscript.

\section{COMPETING INTERESTS}

AR, SO, PF, VT, EB, CA, FM, GM, AC, SF, CC, BC, SM, MA: nothing to disclose; UV: Consulting or advisory role for Celgene, Janssen, Genmab, Incyte, Bristol-Myers Squibb; speakers' bureau with Roche, Celgene, Janssen, Gilead Sciences, Sandoz, Abbvie; research funding from Roche and Celgene; research funding from Roche and Celgene. FB: research funding, Gilead Sciences, Menarini, Emercell. PLZ: speakers' bureau or advisory boards for Verastem, Celltrion, Gilead, Janssen-cilag, Bristol-Myers Squibb, Servier, Sandoz, MSD, Immune Design, Celgene, Portola, Roche, Eusapharma, Kyowa Kirin, Sanofi. PGP: member of advisory commette BRIC Copenaghen, MD Anderson Cancer Center, Houston (Tx). SP: Advisory Boards for Celgene, NanoString, Roche. CT: advisory board, ADC- Therapeutics. ED: research funding from TG-Therapeutics, ADCTherapeutics, Takeda; Advisory board for Gilead, Astra Zeneca, Takeda.

\section{ADDITIONAL INFORMATION}

Supplementary information The online version contains supplementary material available at https://doi.org/10.1038/s41375-021-01347-6.

Correspondence and requests for materials should be addressed to C.T. or E.D.

Reprints and permission information is available at http://www.nature.com/ reprints

Publisher's note Springer Nature remains neutral with regard to jurisdictional claims in published maps and institutional affiliations.

\begin{abstract}
Open Access This article is licensed under a Creative Commons Attribution 4.0 International License, which permits use, sharing, adaptation, distribution and reproduction in any medium or format, as long as you give appropriate credit to the original author(s) and the source, provide a link to the Creative Commons license, and indicate if changes were made. The images or other third party material in this article are included in the article's Creative Commons license, unless indicated otherwise in a credit line to the material. If material is not included in the article's Creative Commons license and your intended use is not permitted by statutory regulation or exceeds the permitted use, you will need to obtain permission directly from the copyright holder. To view a copy of this license, visit http://creativecommons. org/licenses/by/4.0/.
\end{abstract}

(c) The Author(s) 2021 